Article

\title{
An Improved Spatial and Temporal Reflectance Unmixing Model to Synthesize Time Series of Landsat-Like Images
}

\author{
Jianhang Ma ${ }^{1,2}$, Wenjuan Zhang ${ }^{1, *}$, Andrea Marinoni ${ }^{3}$, Lianru Gao ${ }^{1,4}\left(\mathbb{D}\right.$ and Bing Zhang ${ }^{1,2, *(D)}$ \\ 1 Key Laboratory of Digital Earth Science, Institute of Remote Sensing and Digital Earth, \\ Chinese Academy of Sciences, Beijing 100094, China; majh@radi.ac.cn (J.M.); gaolr@radi.ac.cn (L.G.) \\ 2 University of Chinese Academy of Sciences, Beijing 100049, China \\ 3 Centre for Integrated Remote Sensing and Forecasting for Arctic Operations (CIRFA), \\ Department of Physics and Technology, UiT-The Arctic University of Norway, Sykehusvegen 21, \\ NO-9019 Tromsø, Norway; andrea.marinoni@uit.no \\ 4 College of Computer Science and Software Engineering, Computer Vision Research Institute, \\ Shenzhen University, Shenzhen 518060, China \\ * Correspondence: zhangwj@radi.ac.cn (W.Z.); zb@radi.ac.cn (B.Z.)
}

Received: 25 July 2018; Accepted: 28 August 2018; Published: 31 August 2018

\begin{abstract}
The trade-off between spatial and temporal resolution limits the acquisition of dense time series of Landsat images, and limits the ability to properly monitor land surface dynamics in time. Spatiotemporal image fusion methods provide a cost-efficient alternative to generate dense time series of Landsat-like images for applications that require both high spatial and temporal resolution images. The Spatial and Temporal Reflectance Unmixing Model (STRUM) is a kind of spatial-unmixing-based spatiotemporal image fusion method. The temporal change image derived by STRUM lacks spectral variability and spatial details. This study proposed an improved STRUM (ISTRUM) architecture to tackle the problem by taking spatial heterogeneity of land surface into consideration and integrating the spectral mixture analysis of Landsat images. Sensor difference and applicability with multiple Landsat and coarse-resolution image pairs (L-C pairs) are also considered in ISTRUM. Experimental results indicate the image derived by ISTRUM contains more spectral variability and spatial details when compared with the one derived by STRUM, and the accuracy of fused Landsat-like image is improved. Endmember variability and sliding-window size are factors that influence the accuracy of ISTRUM. The factors were assessed by setting them to different values. Results indicate ISTRUM is robust to endmember variability and the publicly published endmembers (Global SVD) for Landsat images could be applied. Only sliding-window size has strong influence on the accuracy of ISTRUM. In addition, ISTRUM was compared with the Spatial Temporal Data Fusion Approach (STDFA), the Enhanced Spatial and Temporal Adaptive Reflectance Fusion Model (ESTARFM), the Hybrid Color Mapping (HCM) and the Flexible Spatiotemporal DAta Fusion (FSDAF) methods. ISTRUM is superior to STDFA, slightly superior to HCM in cases when the temporal change is significant, comparable with ESTARFM and a little inferior to FSDAF. However, the computational efficiency of ISTRUM is much higher than ESTARFM and FSDAF. ISTRUM can to synthesize Landsat-like images on a global scale.
\end{abstract}

Keywords: spatiotemporal image fusion; spatial-unmixing; Improved Spatial and Temporal Reflectance Unmixing Model (ISTRUM); landsat; Substrate, Vegetation, and Dark surface (SVD) linear mixture model 


\section{Introduction}

Landsat images have the longest continuous record of the Earth surface with fine resolution of $30 \mathrm{~m}$ (denoted by fine-resolution image henceforth). It has been widely applied by the scientific community [1]. However, due to the trade-off between spatial and temporal resolution, Landsat sensor revisits the same region every 16 days, which limits its applicability in studies. For example, fine-resolution vegetation phenology mapping [2] and crop type mapping [3] require both high spatial and temporal resolution images. For regions with frequent cloud contamination, the time interval may be longer to achieve an image that can be used to extract information on the instantaneous field of view [4]. To overcome this constraint, spatiotemporal image fusion methods have been developed to synthesize time series of Landsat-like images by blending low spatial resolution but high temporal resolution images (henceforth denoted by coarse-resolution image) such as the MODerate resolution Imaging Spectroradiometer (MODIS) and the Medium Resolution Imaging Spectrometer (MERIS) [5-12]. In the remote sensing image fusion domain, spatiotemporal fusion is different from the superresolution methods which enhance the spatial resolution by combining single-frame or multi-frame images [13], and different from the pansharpening methods (referred to as spatial-spectral fusion) which enhance the spatial resolution and retain spectral resolution of multi-spectral bands with a simultaneously acquired panchromatic band [14]. Spatiotemporal fusion methods aim to generate fine-resolution images with frequent temporal coverage [15]. Inputs of these methods are one or more Landsat and coarse-resolution image pairs (L-C pairs) observed on the same dates (henceforth denoted by base date, $T_{B}$ ), and one coarse-resolution image observed on the prediction date $\left(T_{P}\right)$. Output is a Landsat-like image on $T_{P}$, which captures the temporal change during $T_{B}$ and $T_{P}$ and retains the spatial details. The synthetic time series Landsat-like images have been effectively applied in vegetation monitoring [16,17], crop types mapping [18], fine-resolution land surface temperature monitoring $[19,20]$, daily evapotranspiration mapping [21,22], and water environment monitoring $[23,24]$.

Typically, in technical literature, the spatiotemporal image fusion methods can be categorized into five types [15]: spatial-unmixing based, weight-function based, learning based, Bayesian based, and hybrid methods. The method proposed by Zurita-Milla et al. [6], the STDFA [25] and the STRUM [26] are typical spatial-unmixing based methods. The theoretical basis of these methods is linear spectral mixture of coarse pixels [6]. Different from the spectral-unmixing, which computes the abundance with known spectra of endmembers, spatial-unmixing computes the spectra of endmembers with known abundance. Spatial-unmixing based methods have the advantage of retrieving accurate spectra of endmembers [27]. However, their shortcoming is the synthetic image lacks intra-class spectral variability and spatial details [27]. Because the methods assume each fine pixel contains only one class type and the computed spectra of endmembers are directly assigned to a class image to synthesize the fine-resolution image [6]. The spatial and temporal adaptive reflectance fusion model (STARFM) [5], the Enhanced STARFM (ESTARFM) [7], and the spatial and temporal nonlocal filter-based fusion model (STNLFFM) [28] are typical weight-function based methods. A fine pixel value is estimated by weighting the information of its surrounding similar pixels in these methods. Spatial details are better retained than spatial-unmixing based methods. However, the spectral mixture of coarse pixels are not taken into account in the weighted model [27]. Learning-based methods generally contain two processes, i.e., the learning and applying of the model [15]. Dictionary-pair learning [8,9], regression tree [29], and deep convolutional network [30] have been adopted in learning-based methods with good performance. However, selection of learning samples has significant influence on the accuracy of learning-based methods, and the computation expense is also high to train a model [31]. Some relatively simple but efficient learning-based methods were also proposed. Hazaymeh and Hassan [32,33] proposed a spatiotemporal image-fusion model (STI-FM) which builds the relationship between coarse-resolution images observed on $T_{B}$ and $T_{P}$, and applies the model to Landsat image on $T_{B}$ to synthesize Landsat-like image. STI-FM is capable of enhancing the temporal resolution of both land surface temperature and reflectance images of Landsat-8. Kwan et al. [34,35] 
proposed the HCM which first learns a pixel-to-pixel transformation matrix based on coarse-resolution images and then applies the matrix to Landsat image. Both STI-FM and HCM are simple and computationally efficient with high performance [34]. Bayesian based methods synthesize images in a probabilistic manner based on the Bayesian estimation theory. By making use of the advantage of multivariate arguments, it is flexible to predict temporal changes [36]. However, fusion accuracy is sensitive to the model assumptions and parameter estimations. Spectral mixture of coarse pixels is also not fully incorporated in the methods [36]. By combining advantages of two or more methods of the above four categories, hybrid methods were proposed [15]. The FSDAF [10] is a typical hybrid method. It performs temporal and spatial predictions by spatial-unmixing and Thin Plate Spline (TPS) interpolator separately, and combines two predictions with the idea of weight-function based methods. Hybrid methods could be more flexible in dealing with complicated scenarios, e.g., heterogeneous landscapes [37], abrupt land cover type changes [10] and shape changes [12]. However, accuracy improvement is at the cost of complicated processing steps and computational expense.

Spatiotemporal image fusion methods should capture temporal change of land surface. Because land surface changes in different ways across different regions and periods, each fusion method may have its own suitable situations [26]. There is probably no universally best method. Simple methods can outperform complicated methods if the conditions are suitable [38]. It is valuable to diversify the fusion methods to give more choices in applications [34]. It is also meaningful to improve existing fusion methods. Aiming to fix the shortcoming of the spatial-unmixing based method proposed by Zurita-Milla et al. [6], Gevaert and García-Haro [26] proposed STRUM. Different from the method proposed by Zurita-Milla et al. [6] which directly performs the spatial-unmixing to coarse-resolution image on $T_{P}$, STRUM first calculates a coarse-resolution temporal change image by subtracting coarse-resolution image on $T_{B}$ from the image on $T_{P}$. Then, the difference image is disaggregated by spatial-unmixing to synthesize the fine-resolution temporal change image, which is further added to Landsat image on $T_{B}$ to synthesize Landsat-like image on $T_{P}$. Therefore, the synthetic image inherits spectral variability and spatial details from Landsat image on $T_{B}$ to some degree [26]. The STRUM outperforms STARFM and original spatial-unmixing method, even when there are few reference Landsat images [26]. However, because classification image is applied in spatial-unmixing process, the downscaled fine-resolution temporal change image in STRUM still lacks intra-class spectral variability and spatial details.

Because of the heterogeneity of land surface, more than one land cover types could exist in a Landsat pixel at the scale of $30 \mathrm{~m}$. Mixed pixels commonly occur in Landsat image [39]. The hard-classification image is unable to represent the varied combinations of endmembers in the pixels [39]. Generally, land surface varies gradually even on the boundaries of different land cover types. The abundance image, which records area fractions of endmembers in the pixel, could report continuous gradations and retain the spatial structure of land surface, thus providing a more accurate representation of land surface than class image [39]. Therefore, it is more appropriate to mix spectra of endmembers with fine-resolution abundance image than to assign it to classification image. For the reasons above, we proposed Improved STRUM (ISTRUM) by taking advantages of abundance images in order to tackle the aforementioned shortcoming of STRUM. By surveying the studies on the spatial-unmixing based methods $[6,25,26,40,41]$, we found that ISTRUM is the first study that applies fine-resolution abundance image in spatial-unmixing process. In STRUM, the derived fine-resolution temporal change image based on coarse-resolution image is directly added to Landsat image. The sensor difference between Landsat and coarse-resolution imaging systems is not considered [26]. It was adjusted with a linear model in ISTRUM. Moreover, STRUM only considered the situation when only one L-C image pair is available [26]. A method to combine results synthesized by multiple L-C pairs was integrated in ISTRUM in order to enhance its applicability for situations when multiple L-C pairs are available.

The objective of this paper is: (1) to describe the framework of ISTRUM; (2) to evaluate the performance improvement of ISTRUM by comparing it with STRUM; (3) to analyze the sensitivity of 
ISTRUM to factors that influence the accuracy; and (4) to compare the performance of ISTRUM with STDFA, ESTARFM, HCM and FSDAF.

\section{Prediction Method}

ISTRUM aims to synthesize time series of Landsat-like images based on at least one L-C pair observed on $T_{B}$, and a coarse-resolution image observed on $T_{P}$. Similar to other spatiotemporal fusion methods [5,6,26], all input images should be atmospherically corrected and geometrically co-registered. The coarse-resolution images should contain similar spectral bands of Landsat images.

\subsection{Notations and Definitions}

The coarse-resolution images observed on $T_{B}$ and $T_{P}$ are stored in array $\mathbf{C}^{B}$ and $\mathbf{C}^{P}$, respectively. Dimensions of $\mathbf{C}^{B}$ and $\mathbf{C}^{P}$ are $n_{c x} \times n_{c y} \times n_{b}$, where $n_{c x}$ and $n_{c y}$ denote the lines and samples of the coarse-resolution images and $n_{b}$ denotes spectral bands. The Landsat image observed on $T_{B}$ and $T_{P}$ is stored in array $\mathbf{F}^{B}$ and $\mathbf{F}^{P}$, respectively. The synthetic Landsat-like image based on L-C pair on $T_{B}$ is stored in $\mathbf{F}^{P_{-} B}$. Dimensions of $\mathbf{F}^{B}, \mathbf{F}^{P}$, and $\mathbf{F}^{P-B}$ are $n_{f x} \times n_{f y} \times n_{b}$, where $n_{f x}$ and $n_{f y}$ denote the lines and samples of the Landsat images. The spatial resolution ratio is $S=n_{f x} / n_{c x}$.

A pixel is selected according to its sample, line, and band indices which are denoted by $i, j$, and $b$, respectively. For example, $\left[i_{c}, j_{c}, b\right]$ denotes a coarse pixel at location $\left[i_{c}, j_{c}\right]$ of band $b$, where $i_{c} \in\left\{1,2, \ldots, n_{c x}\right\}, j_{c} \in\left\{1,2, \ldots, n_{c y}\right\}$ and $b \in\left\{1,2, \ldots, n_{b}\right\}$. Subscript $c$ and $f$ denot coarse- and fine-resolution images, respectively. For all valid sample, line, or band indices of an image, we use the asterisk notation. For example, $\left[i_{c}, j_{c}, *\right]$ refers to all band values for pixel at location $\left[i_{c}, j_{c}\right]$, and $[*, *, b]$ refers to all pixels of band $b$. To select pixels in a sliding-window from the image, we use $\left[i_{1 c}: i_{2 c}, j_{1 c}: j_{2 c}, b\right]$ to denote the subset covers lines $i_{1 c}, \ldots, i_{2 c}$ and samples $j_{1 c}, \ldots, j_{2 c}$ of band $b$. There are $S^{2}$ fine pixels within a coarse pixel at location $\left[i_{c}, j_{c}\right]$. For $k$-th fine pixel, its location on the fine-resolution image is denoted as $\left[k_{i c}, k_{j c}\right]$ with $k \in\left\{1,2, \ldots, S^{2}\right\}$.

\subsection{Introduction of STRUM}

STRUM generally contains the following steps [26]: (1) Define the number of classes on image $\mathbf{F}^{B}$ and perform classification to obtain a fine-resolution class image. The class types are considered as endmembers of coarse-resolution image. (2) Calculate a coarse-resolution abundance image with the class image. For a coarse pixel, abundance of each endmember is the ratio of the number of fine pixels with corresponding class type to $S^{2}$. (3) Calculate coarse-resolution temporal change image $\Delta \mathbf{C}$ by $\mathbf{C}^{P}-\mathbf{C}^{B}$. (4) $\Delta \mathbf{C}$ is assumed to be linear mixture of endmembers and downscaled with spatial-unmixing to obtain a fine-resolution temporal change image $\Delta \mathbf{F}$. The step first calculates spectra of endmembers by solving a system of linear equations which is established by coarse pixels in a sliding-window, then assigns the spectra to fine pixels with corresponding class type. (5) $\mathbf{F}^{P_{-} B}$ is calculated by $\mathbf{F}^{B}+\Delta \mathbf{F}$.

STRUM assumes a fine pixel contains only one class type. Therefore, Step (2) calculates coarse-resolution abundance by counting the number of fine pixels, and Step (4) directly assign spectra of endmembers to fine pixels. It results in the lack of intra-class spectral variability on image $\Delta \mathbf{F}$ because pixels of same class type have same values. In addition, spectra derived by unmixing of $\Delta \mathbf{C}$ have spectral characteristics of coarse-resolution imaging system. Sensor difference should be adjusted before adding $\Delta \mathbf{F}$ to $\mathbf{F}^{B}$.

\subsection{Theoretical Basis and Prediction Model of ISTRUM}

Because of the heterogeneity of land surface, more than one land cover types can exist in both Landsat pixels (e.g., $30 \mathrm{~m} \times 30 \mathrm{~m}$ ) and coarse pixels (e.g., $500 \mathrm{~m} \times 500 \mathrm{~m}$ for MODIS). A linear mixture model could represent the combinations of endmembers' reflectance and abundance [42]. For a Landsat pixel $\left[i_{f}, j_{f}, b\right]$, its reflectance on $T_{B}$ and $T_{P}$ are 


$$
\begin{aligned}
& \mathbf{F}^{B}\left[i_{f}, j_{f}, b\right]=\sum_{m=1}^{n_{m}} \mathbf{A}_{F}^{B}\left[i_{f}, j_{f}, m\right] \times \mathbf{E}_{F}^{B}[m, b]+\mathcal{E}\left[i_{f}, j_{f}, b\right] \\
& \mathbf{F}^{P}\left[i_{f}, j_{f}, b\right]=\sum_{m=1}^{n_{m}} \mathbf{A}_{F}^{P}\left[i_{f}, j_{f}, m\right] \times \mathbf{E}_{F}^{P}[m, b]+\mathcal{E}\left[i_{f}, j_{f}, b\right]
\end{aligned}
$$

where $n_{m}$ is the number of endmembers and $m$ denotes the $m$-th endmember with $m \in\left\{1,2, \ldots, n_{m}\right\}$. $\mathbf{A}_{F}^{B}$ and $\mathbf{A}_{F}^{P}$ are fine-resolution abundance images on $T_{B}$ and $T_{P}$ with dimensions of $n_{f x} \times n_{f y} \times n_{m}$. $\mathbf{E}_{F}^{B}$ and $\mathbf{E}_{F}^{P}$ are $n_{m} \times b$ arrays that store the reflectance of endmembers on $T_{B}$ and $T_{P} . \mathcal{E}$ is the residual. Assuming abundance images do not change between $T_{B}$ and $T_{P}$, i.e., $\mathbf{A}_{F}^{B}=\mathbf{A}_{F}^{P}$, and $\mathcal{E}$ is constant, we have

$$
\Delta \mathbf{F}\left[i_{f}, j_{f}, b\right]=\sum_{m=1}^{n_{m}} \mathbf{A}_{F}^{B}\left[i_{f}, j_{f}, m\right] \times \Delta \mathbf{E}_{F}[m, b]
$$

with

$$
\begin{gathered}
\Delta \mathbf{F}=\mathbf{F}^{P}-\mathbf{F}^{B} \\
\Delta \mathbf{E}_{F}=\Delta \mathbf{E}_{F}^{P}-\Delta \mathbf{E}_{F}^{B}
\end{gathered}
$$

where $\Delta \mathbf{F}$ denotes the temporal change of fine-resolution images and $\Delta \mathbf{E}_{F}$ denotes the reflectance change of endmembers on fine-resolution images. Among the variables, only $\mathbf{F}^{B}$ is known and $\mathbf{A}_{F}^{B}$ could be derived from $\mathbf{F}^{B}$ by spectral-unmixing. If $\Delta \mathbf{E}_{F}$ were obtained, $\Delta \mathbf{F}$ would be calculated by linear mixture (Equation (3)) and a Landsat-like image would be predicted by adding $\Delta \mathbf{F}$ to $\mathbf{F}^{B}$ (Equation (4)).

Similarly with Equations (3)-(5), temporal change of coarse-resolution images $\Delta \mathbf{C}$ can be expressed as

$$
\Delta \mathbf{C}\left[i_{c}, j_{c}, b\right]=\sum_{m=1}^{n_{m}} \mathbf{A}_{C}^{B}\left[i_{c}, j_{c}, m\right] \times \Delta \mathbf{E}_{C}[m, b]
$$

with

$$
\begin{gathered}
\Delta \mathbf{C}=\mathbf{C}^{P}-\mathbf{C}^{B} \\
\Delta \mathbf{E}_{C}=\Delta \mathbf{E}_{C}^{P}-\Delta \mathbf{E}_{C}^{B}
\end{gathered}
$$

where $\mathbf{A}_{C}^{B}$ denotes the coarse-resolution abundance image with dimensions of $n_{c x} \times n_{c y} \times n_{m} . \mathbf{E}_{C}^{B}$ and $\mathbf{E}_{C}^{P}$ are $n_{m} \times b$ arrays that identify the reflectance of endmembers on coarse-resolution image, and $\Delta \mathbf{E}_{C}$ represents the reflectance change of the endmembers on coarse-resolution images.

Among the variables in Equations (6)-(8), $\mathbf{C}^{P}$ and $\mathbf{C}^{B}$ are known and $\Delta \mathbf{C}$ could be calculated. Moreover, because the types and spatial distributions of endmembers should be same for fine- and coarse-resolution images in same regions, $\mathbf{A}_{C}^{B}$ can be aggregated from $\mathbf{A}_{F}^{B}$ with

$$
\mathbf{A}_{C}^{B}\left[i_{c}, j_{c}, m\right]=\frac{1}{S^{2}} \sum_{k=1}^{S^{2}} \mathbf{A}_{F}^{B}\left[k_{i c}, k_{j c}, m\right]
$$

Equation (9) indicates the abundance of $m$-th endmember in a coarse pixel $\left[i_{c}, j_{c}\right]$ is calculated by averaging the abundances of $m$-th endmember in the corresponding fine pixels. However, in STRUM, $\mathbf{A}_{C}^{B}\left[i_{c}, j_{c}, m\right]$ is the ratio of the count of fine pixels for class $m$ to $S^{2}$ [26].

Although $\Delta \mathbf{C}\left[i_{c}, j_{c}, b\right]$ and $\mathbf{A}_{C}^{B}\left[i_{c}, j_{c}, *\right]$ of Equation (6) are known variables, $\Delta \mathbf{E}_{C}$ is unsolvable because Equation (6) is underdetermined since there are $n_{m}$ unknowns of $\Delta \mathbf{E}_{C}[*, b]$ [6].

According to Waldo Tobler's first law of geography [43], reflectance of same land cover type in a small region can change similarly over time, e.g., the maize in adjacent crop fields may grow in 
similar ways and have analogous spectral features. Therefore, it is reasonable to assume $\Delta \mathbf{E}_{C}[m, *]$ is same in a sliding-window of coarse-resolution image. With half of the sliding-window size $w_{h}$, which is measured by the number of coarse pixels and should be defined by user, a sliding-window centred at pixel $\left[i_{c}, j_{c}\right]$ is selected by $\left[\left(i_{c}-w_{h}\right):\left(i_{c}+w_{h}\right),\left(j_{c}-w_{h}\right):\left(j_{c}+w_{h}\right)\right]$. Size of sliding-window is $w=2 \times w_{h}+1$. Then, $w^{2}$ linear equations similar with Equation (6) are established with the coarse pixels. To derive $\Delta \mathbf{E}_{C}[*, b]$ by solving the linear equations, $w^{2}$ should be greater than $n_{m}$ [6]. Given the reflectance change is also related to environmental factors (e.g., altitude, morphology, soil type, and fertilization) [40], the assumption that $\Delta \mathbf{E}_{C}[m, *]$ is the same may be valid only in a small region. Therefore, the definition of $w_{h}$ should take spatial resolution of the coarse-resolution image and heterogeneity of land surface into consideration. $w_{h}$ should not be too large, otherwise, it may induce errors in the calculation of $\Delta \mathbf{E}_{C}[m, *]$.

Theoretically, reflectance of endmembers on Landsat and coarse-resolution images should also be the same. However, sensor difference, caused by differences between fine- and coarse-resolution sensor systems such as bandwidth, solar-viewing geometry conditions, atmospheric correction, and surface reflectance anisotropy [44,45] may exist. The computation of $\Delta \mathbf{E}_{C}$ is based on $\Delta \mathbf{C}$. Thus, it has spectral characteristics of coarse-resolution imaging system. To obtain $\Delta \mathbf{E}_{F}$, sensor difference should be adjusted. Linear and nonlinear models have been proposed to normalize the difference between images of different sensors [46]. Similar to previous studies [47,48], a simple linear model is applied to adjust sensor difference.

$$
\mathbf{E}_{F}^{T}[*, b]=a_{l}[b] \times \mathbf{E}_{C}^{T}[*, b]+b_{l}[b], \text { with } T=B \text { or } P
$$

In Equation (10), $a_{l}[b]$ and $b_{l}[b]$ are slope and interception of the linear model for band $b$ and can be calculated by linear regression between the observed fine- and coarse-resolution images. Sensor difference between $\Delta \mathbf{E}_{F}$ and $\Delta \mathbf{E}_{C}$ can be adjusted by Equation (11). The $b_{l}[b]$ in Equation (10) is reduced after subtraction.

$$
\Delta \mathbf{E}_{F}[*, b]=a_{l}[b] \times \Delta \mathbf{E}_{C}[*, b]
$$

In STRUM, $\Delta \mathbf{E}_{F}$ is directly assigned to fine pixels with same class type according to a fine class image [26]. However, $\Delta \mathbf{E}_{F}$ is linearly mixed with $\mathbf{A}_{F}^{B}$ in ISTRUM. Since $\Delta \mathbf{E}_{F}$ is the local reflectance change of the endmembers, the mixture is only performed on fine pixels that fall in the central coarse pixel. The $\Delta \mathbf{F}$ is calculated as follow:

$$
\Delta \mathbf{F}\left[k_{i c}, k_{j c}, b\right]=\sum_{m=1}^{n_{m}} \mathbf{A}_{F}^{B}\left[k_{i c}, k_{j c}, m\right] \times \Delta \mathbf{E}_{F}[m, b]
$$

Based on one L-C pair, the Landsat-like image on $T_{P}$ is finally calculated by

$$
\mathbf{F}^{P \_B}=\mathbf{F}^{B}+\Delta \mathbf{F}
$$

The aforesaid steps describe the prediction model with one L-C pair. When multiple L-C pairs are applied, $\mathbf{F}^{P \_} B$ could be first predicted based on each L-C pair and then combined to make a final prediction.

\subsection{Implementation of ISTRUM}

The workflow of ISTRUM is outlined by the flowchart in Figure 1. Key steps (steps with gray background) include spectral-unmixing, abundance aggregation, spatial-unmixing, sensor difference adjustment and linear mixture. Spectral-unmixing is the first step aiming to derive fine-resolution abundance image $\mathbf{A}_{F}^{B}$ which is used in abundance aggregation and linear mixture. Based on $\mathbf{A}_{F}^{B}$, coarse-resolution abundance $\mathbf{A}_{C}^{B}$ is calculated in abundance aggregation. With known $\mathbf{A}_{C}^{B}$ and coarse-resolution temporal change image $\Delta \mathbf{C}$, reflectance change of endmembers are calculated in spatial-unmixing process. Derived $\Delta \mathbf{E}_{C}$ has spectral characteristics of coarse-resolution imaging 
system. Sensor difference is adjusted to obtain $\Delta \mathbf{E}_{F}$, which is further linear mixed with $\mathbf{A}_{F}^{B}$ to obtain fine-resolution temporal change image. Detailed descriptions are in the following subsections.

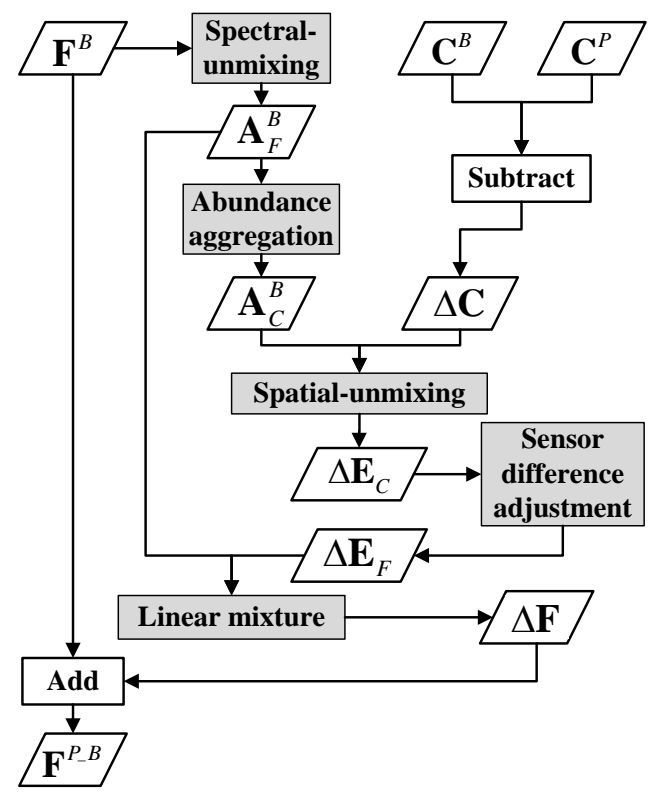

Figure 1. Flowchart of the ISTRUM.

\subsubsection{Spectral-Unmixing}

Spectral-unmixing is the first step applied to $\mathbf{F}^{B}$ to obtain $\mathbf{A}_{F}^{B}$ which is further used to calculate $\mathbf{A}_{C}^{B}$. To strengthen the applicability of ISTRUM on the global scale, a globally representative spectral linear mixture model (SVD model) for Landsat TM/ETM+/OLI images $[39,49,50]$ was selected in the implementation of spectral-unmixing. Endmembers were classified to three types: substrate (S), vegetation $(\mathrm{V})$, and dark surface $(\mathrm{D})$.

Spectra of endmembers (i.e., $\mathbf{E}_{F}^{B}$ ) are extracted from $\mathbf{F}^{B}$ with the approach described by Small [39]. The approach first applies Principal Component Analysis (PCA) to $\mathbf{F}^{B}$, and then selects pure pixels by analyzing the mixing space of the first three primary components. The approach generally selects a suite of pure pixels for each endmember and mean spectra are used in spectral-unmixing. Based on the mixture model, the Fully Constrained Least Square (FCLS) method [51] is used to unmix $\mathbf{F}^{B}$ in the spectral-unmixing process. The computed abundance $\mathbf{A}_{F}^{B}$ varied from 0 to 1 and the sum of $\mathbf{A}_{F}^{B}\left[i_{f}, j_{f}, *\right]$ is 1 .

\subsubsection{Abundance Aggregation}

$\mathbf{A}_{C}^{B}$ is calculated with $\mathbf{A}_{F}^{B}$ in this process. For a coarse pixel at location $\left[i_{c}, j_{c}\right]$, abundance of each endmember is calculated by Equation (9). If an endmember abundance is low in a coarse pixel, its errors in spatial-unmixing may be large [6]. Therefore, if the abundance of endmember $m$ is $0<\mathbf{A}_{C}^{B}\left[i_{c}, j_{c}, m\right]<t h_{A}$, it is merged to its spectrally most similar class whose abundance should also be greater than 0 . In the implementation of ISTRUM, similarity between each endmember is measured by Spectral Angle (SA), which is calculated with $\mathbf{E}_{F}^{B}$. $t h_{A}$ is set to 0.05 according to previous studies $[6,52]$.

\subsubsection{Spatial-Unmixing}

The spatial-unmixing process aims to derive $\Delta \mathbf{E}_{C}$ by solving a system of linear equations. For a coarse pixel at location $\left[i_{c}, j_{c}\right]$, a sliding-window around it is selected by $\left[\left(i_{c}-w_{h}\right):\left(i_{c}+w_{h}\right)\right.$, $\left.\left(j_{c}-w_{h}\right):\left(j_{c}+w_{h}\right)\right]$ and $w^{2}$ equations similar with Equation (6) are considered. The least square method is applied to solve the equations because it does not require additional parameters and 
has been widely implemented in spatial-unmixing based methods $[6,25,48] . \Delta \mathbf{E}_{C}$ is computed by minimizing Equation (14) based on the Generalized Reduced Gradient Method [53] as follow:

$$
\Delta \mathbf{E}_{C}[*, b]=\arg \min \left\{\sum_{i=i_{c}-w_{h}}^{i_{c}+w_{h}} \sum_{j_{c}-w_{h}}^{j_{c}+w_{h}}\left(\Delta \mathbf{C}[i, j, b]-\sum_{m=1}^{n_{m}} \mathbf{A}_{C}^{B}[i, j, m] \times \Delta \mathbf{E}_{C}[m, b]\right)^{2}\right\}
$$

\subsubsection{Sensor Difference Adjustment}

Sensor difference is adjusted to obtain $\Delta \mathbf{E}_{F}$ from $\Delta \mathbf{E}_{C}$ by means of Equation (11). The coefficient $a_{l}$ is calculated with $\mathbf{F}^{B}$ and $\mathbf{C}^{B} . \mathbf{F}^{B}$ is firstly aggregated to coarse-resolution image $\mathbf{F}_{a}^{B}$ by averaging the band values of fine pixels that fall in an individual coarse pixel. Then, $a_{l}[b]$ is computed by linear regression with $\mathbf{F}_{a}^{B}[*, *, b]$ as dependent variable and $\mathbf{F}^{C}[*, *, b]$ as independent variable.

\subsubsection{Linear Mixture}

The $\Delta \mathbf{E}_{F}$ is linearly mixed with $\mathbf{A}_{F}^{B}$ (Equation (12)) to reconstruct $\Delta \mathbf{F}$. The mixture is only applied to fine pixels (i.e., $\left[k_{i c}, k_{j c}\right]$ ) falling in the center coarse pixel $\left[i_{c}, j_{c}\right]$ of the currently selected sliding-window. Because $\Delta \mathbf{E}_{F}$ represents the reflectance change of endmembers in the sliding-window.

The spatial-unmixing and linear mixture processes are performed pixel-by-pixel for each band of $\Delta \mathbf{C}$. When all the pixels of $\Delta \mathbf{C}$ are processed, image $\Delta \mathbf{F}$ is obtained and the Landsat-like image $\mathbf{F}_{-}^{P} B$ predicted by one L-C pair is finally calculated by Equation (13).

\subsubsection{Method to Combine Images Predicted by Multiple L-C Pairs}

Suppose there are $n_{T}$ L-C pairs observed on different base dates $T_{B}$ with $B \in\left\{1,2, \ldots, n_{T}\right\}$. Each L-C pair could synthesize an individual Landsat-like image $\mathbf{F}_{-}^{P_{-} B}$. The final prediction is weighted summation of all the $\mathbf{F}^{P_{-} B}$. Absolute temporal difference $\left(D_{i j b}^{B}\right)$ between $T_{P}$ and $T_{B}$ is used to calculate the weights. $D_{i j b}^{B}$ is calculated locally by Equation (15) with $\Delta \mathbf{C}$ in a sliding-window. The window is same with the one applied in spatial-unmixing because reflectance change of each endmember is assumed to be same in the sliding-window. When $D_{i j b}^{B}$ value approximates 0 , it indicates little reflectance change between $T_{B}$ and $T_{P}$, so the prediction based on L-C pair on date $T_{B}$ should be more reliable. Therefore, $\mathbf{F}^{P}{ }_{-}^{B}$ should have a large weight. Hence, weight $W_{i j b}^{B}$ is calculated with the reciprocal of $D_{i j b}^{B}$ in Equation (16). The $W_{i j b}^{B}$ is for the central coarse pixel $\left[i_{c}, j_{c}\right]$ of the sliding-window, and fine pixels within it share the same weight. The final prediction $\left(\mathbf{F}^{P}{ }^{M}\right)$ with multiple L-C pairs is calculated by Equation (17).

$$
\begin{gathered}
D_{i j b}^{B}=\sum_{i=i_{c}-w_{h}}^{i_{c}+w_{h}} \sum_{j=j_{c}-w_{h}}^{j_{c}+w_{h}}|\Delta \mathbf{C}[i, j, b]| \\
W_{i j b}^{B}=\frac{1 / D_{i j b}^{B}}{\sum_{B=1}^{n_{T}} 1 / D_{i j b}^{B}} \\
\mathbf{F}^{P_{-} M}\left[k_{i c}, k_{j c}, b\right]=\sum_{B=1}^{n_{T}} W_{i j b}^{B} \times \mathbf{F}^{P_{-} B}\left[k_{i c}, k_{j c}, b\right]
\end{gathered}
$$

\subsection{Differences between ISTRUM and STRUM}

Both ISTRUM and STRUM applied spatial-unmixing process directly to the coarse-resolution temporal change image. However, there are some differences. ISTRUM considers the heterogeneity of land surface and regards the pixels on Landsat image are linear mixture of endmembers at the scale of $30 \mathrm{~m}$. Therefore, the fine-resolution abundance image $\mathbf{A}_{F}^{B}$ is applied in ISTRUM, while class image is used in STRUM. Based on the difference, the $\Delta \mathbf{E}_{F}$ is further linearly mixed with $\mathbf{A}_{F}^{B}$ to obtain $\Delta \mathbf{F}$. 
The step ensures $\Delta \mathbf{F}$ to better preserve spectral variability and spatial details than the one derived by STRUM. In addition, sensor difference between $\Delta \mathbf{E}_{F}$ and $\Delta \mathbf{E}_{C}$ is considered and adjusted in ISTRUM. While STRUM directly assigns $\mathbf{E}_{C}$ to fine pixels according to the class image without adjusting sensor difference.

As reference images, the L-C pairs input into the fusion method have significant influence on the prediction accuracy [28,54]. According to [55], two L-C pairs is preferred to be applied in the prediction when temporal change is consistent. ISTRUM can combine prediction results of multiple L-C pairs with a weighted summation method, which strengthens the applicability, while STRUM is applicable with only one L-C pair.

\section{Data and Experiment Schemes}

\subsection{Study Area}

Performance of ISTRUM was evaluated with Landsat and MODIS images of Lower Gwydir Catchment ("Gwydir" henceforth) in northern New South Wales (149.2408 ${ }^{\circ}$, 29.1146 ${ }^{\circ}$ S), Australia, acquired on 16 April, 2 May, 5 July, and 22 August 2004. Day Of Year (DOY) for each image pair is 107, 123, 187, and 235. The images were shared by Emelyanova et al. [38] and have been widely applied in the evaluation of spatiotemporal image fusion methods $[31,38,56]$. The images were atmospherically corrected and geographically co-registered [38]. Pixel size of both Landsat and MODIS images was resampled to $25 \mathrm{~m}$ with nearest neighbor method by Emelyanova et al. [38]. Because the coarse-resolution image should be in its original spatial resolution to be applied in ISTRUM, we aggregated the MODIS pixel to $500 \mathrm{~m}$ resolution. Therefore, spatial resolution ratio $S$ is 20. Dimensions of the Landsat and MODIS images applied in this study are $2000 \times 2000 \times 6$ and $100 \times 100 \times 6$, respectively. The Landsat and MODIS images are shown in Figure 2. Temporal dynamics were mainly caused by the vegetation phenology between DOY107 and DOY235. Images on DOY107 and DOY123 are the most similar pairs because of their short time interval. Vegetation growth from DOY187 to DOY235 leads to the reflectance differences in some regions. Bare soil changed to vegetation in large regions from DOY123 to DOY187.
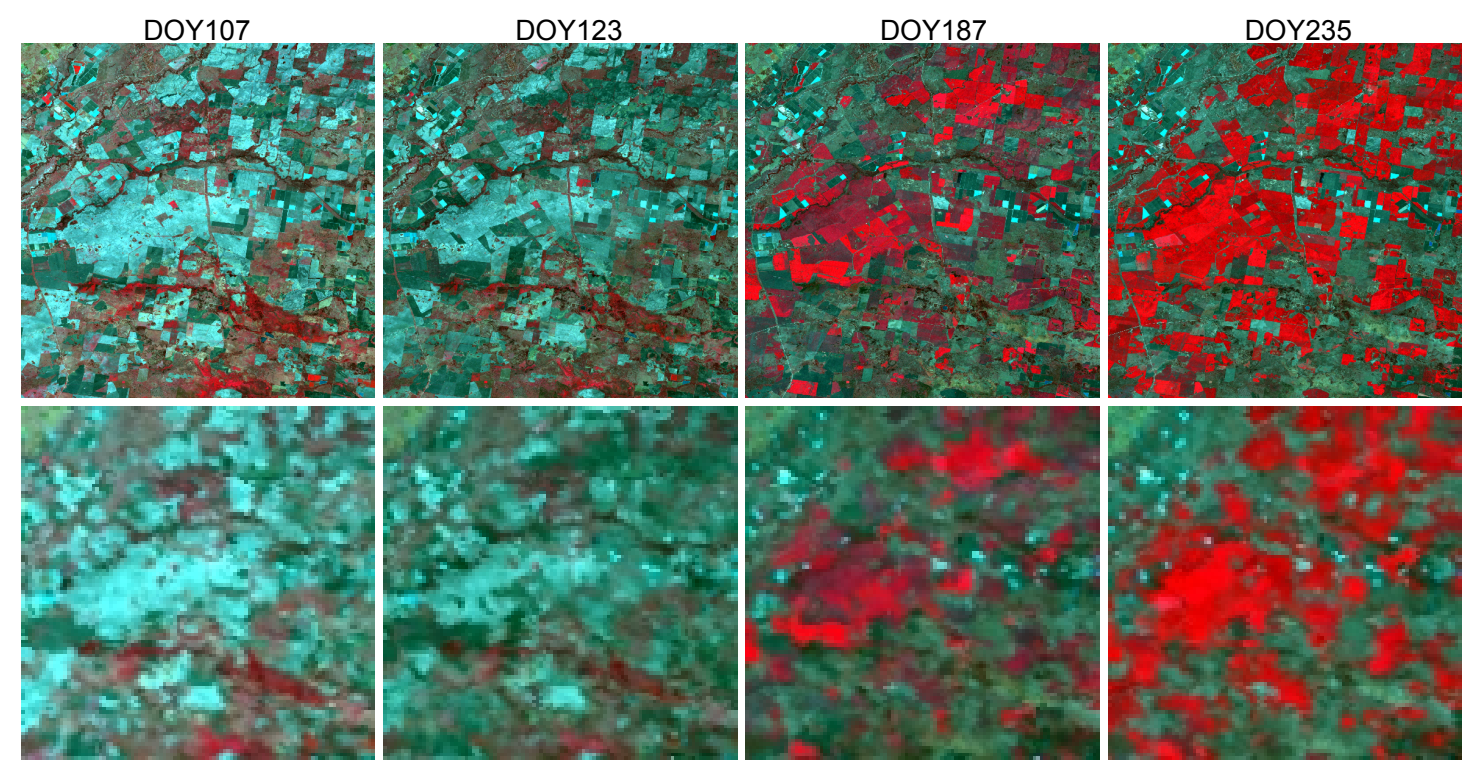

Figure 2. Landsat (first row) and MODIS (second row) images of study area. 


\subsection{Experiment Schemes}

\subsubsection{Comparisons between ISTRUM and STRUM}

The experiment aimed to compare the performance of ISTRUM and STRUM. A Landsat-like image was predicted by ISTRUM and STRUM separately based on one L-C pair on its previous date. For example, the Landsat image on DOY123 was predicted by an L-C pair on DOY107 (DOY123by107). There are three experimental combinations: DOY123by107, DOY187by123, and DOY235by187.

In the experiment, ISTRUM and STRUM were tested by setting user defined parameters to different values for all the combinations. Because SVD model is applied in ISTRUM, $n_{m}$ is 3 and does not need to be defined by user. The parameters required to be defined include $w_{h}$ and $n_{m}$ for STRUM and $w_{h}$ for ISTRUM. $w_{h}$ was set to $\{1,2, \ldots, 10\}$ in step of 1 (i.e., $w \in\{3,5, \ldots, 21\}$ ). $n_{m}$ was set to 3,5 , 7 , and 9 for STRUM. In spectral-unmixing process of ISTRUM, spectra of endmembers were extracted manually with the approach described by Small [39]. To obtain the classification image applied in STRUM, ISODATA was used to classify $\mathbf{F}^{B}$. Observed Landsat images were used to evaluate the prediction accuracy.

Performance of STRUM and ISTRUM under different parameter settings can be compared with results of the experiment. Influence of $w_{h}$ on ISTRUM could also be analyzed. In addition, performance improvements of ISTRUM were evaluated in detail with DOY187by123 because significant temporal change of vegetation occurred during DOY123 and DOY187.

\subsubsection{Sensitivity of ISTRUM to Endmember Variability}

The fine-resolution abundance image $\mathbf{A}_{F}^{B}$ is used to calculate coarse-resolution abundance image which affects the spatial-unmixing process. Derived $\Delta \mathbf{E}_{F}$ is also linearly mixed with $\mathbf{A}_{F}^{B}$ to obtain $\Delta \mathbf{F}$ which directly affects the synthetic image. Therefore, it is necessary to assess whether the uncertainties in $\mathbf{A}_{F}^{B}$ heavily influence accuracy of ISTRUM.

$\mathbf{A}_{F}^{B}$ is obtained by spectral-unmixing of $\mathbf{F}^{B}$. Endmember variability, caused by the temporal and spatial variability of scene components and imaging conditions [57], is the key factor leading to the uncertainties in $\mathbf{A}_{F}^{B}$. Assessment of the influence of $\mathbf{A}_{F}^{B}$ is performed by applying different spectra combinations of endmembers in the spectral-unmixing process. If the accuracy of ISTRUM varies little as the spectra of endmembers varies, it means ISTRUM is robust to endmember variability. Otherwise, ISTRUM is sensitive to endmember variability. Based on this idea, we first determined 27 spectra combinations of endmembers. Each combination was applied to spectral-unmixing process to derive a $\mathbf{A}_{F}^{B}$. The $27 \mathbf{A}_{F}^{B}$ images were separately applied in the prediction of a Landsat-like image with following steps of ISTRUM. Finally, the accuracy of 27 Landsat-like images was calculated and analyzed. Only images on DOY187 and DOY235 were applied in the experiment.

Small and Milesi [49] extracted the spectra of Global SVD endmembers from 100 diverse Landsat TM/ETM+ sub-scenes which were collected globally, and shared the spectra online at http:/ /www. ldeo.columbia.edu/\%7esmall/GlobalLandsat/. Although the spectra of endmembers of a specific study area may be different from the Global SVD spectra, we also tested the applicability of Global SVD spectra in experimental combinations DOY123by107, DOY187by123, and DOY235by187. The Global SVD was applied to derive $\mathbf{A}_{F}^{B}$ in spectral-unmixing process for the prediction of Landsat-like images. Prediction accuracy was compared with results when spectra of endmembers were extracted manually. If the ISTRUM were robust to spectral variability, the Global SVD would be valuable in future application of ISTRUM and only $w_{h}$ should be defined by user. 


\subsubsection{ISTRUM with two L-C Pairs}

To test ISTRUM with two L-C pairs, Landsat-like images on DOY123 and DOY187 were predicted with L-C pairs on DOY107 and DOY235 (i.e., experimental combinations DOY123by107\&235 and DOY187by107\&235). The accuracy was compared with results when one L-C pair was applied.

\subsubsection{Comparisons between ISTRUM and Benchmark Spatiotemporal Fusion Methods}

Performance of ISTRUM was also evaluated by comparing it with benchmark spatiotemporal fusion methods. STDFA, ESTARFM, HCM and FSDAF methods were selected in the comparison. The methods used include spatial-unmixing based, weight-function based, learning based and hybrid methods. STDFA is a spatial-unmixing based method proposed by Wu et al. [25]. It first performs spatial-unmixing process to coarse-resolution image on $T_{B}$ and $T_{P}$ separately. Then, it synthesizes fine-resolution image on $T_{P}$ with a Surface Reflectance Calculation Model (SRCM) [25]. ESTARFM is a weight-function based method and is improved based on STARFM [7]. Since comparison between STRUM and STARFM has been performed in [26], we compared the ISTRUM and ESTARFM in this study because they are both improved methods. In the implementation of ESTARFM, class image was used to identify similar pixels according to [58]. HCM is a learning based method [34,35]. It synthesizes Landsat-like image by applying transformation matrix, which is learned from coarse-resolution images observed on $T_{B}$ and $T_{P}$, to Landsat image observed on $T_{B}$. HCM is a newly developed method and has high performance and computational efficiency [34]. FSDAF is a hybrid method [10]. It considers temporal and spatial variation between $T_{B}$ and $T_{P}$, and it has the ability to predict land cover type change.

To synthesize a Landsat-like image, STDFA, HCM and FSDAF require one L-C pair, while ESTARFM requires two L-C pairs, and ISTRUM is applicable with one or more L-C pairs. To ensure the input images are the same for the methods, experimental combinations DOY123by107, DOY187by123, and DOY235by187 were used in the comparison of STDFA, HCM, FSDAF and ISTRUM. Experimental combinations DOY123by107\&235 and DOY187by107\&235 were used in the comparison of ESTARFM and ISTRUM.

\subsection{Quantitative Evaluation Indices}

Correlation coefficient (CC), relative root mean square error (RRMSE), spectral angle mapper $(\mathrm{SAM})$, and $Q 2^{n}[59]$ between synthetic Landsat-like and observed Landsat image were used to deliver quantitatively assessment of accuracy. The ideal value of $C C$ is 1 and a high value indicates a strong linear relationship between synthetic and observed images. RRMSE is defined as the ratio of the root mean square error of a predicted image to the mean value of the original image and the ratio multiplied by 100 [18]. The ideal value of RRMSE is 0. Low RRMSE indicates small difference in pixel values between synthetic and observed images. Spectral angle with unit of degree between synthesized and observed images was calculated. The ideal value of SAM is 0. Small SAM value indicates high spectral consistency. $Q 2^{n}$ is suitable to assess the spatial structure errors for images with an arbitrary number of bands [59]. The ideal value of $Q 2^{n}$ is 1 . A high $Q 2^{n}$ value indicates high spatial consistency between the synthetic image and observed image. Each band of image has a CC and RRMSE value, while each image has a SAM and $Q 2^{n}$ value in the assessment.

The reduction in remaining error (RRE) [60] was also used to quantify the improvements in accuracy of different methods. RRE is calculated as

$$
R R E=\frac{R E_{1}-R E_{0}}{R E_{1}} \times 100 \%
$$

$R E_{1}$ and $R E_{0}$ are remaining errors of the compared methods and ISTRUM, respectively. For CC and $Q 2^{n}, R E$ is calculated by $1-C C$ and $1-Q 2^{n}$, respectively. For RRMSE and SAM, $R E$ is the calculated RRMSE and SAM value. The positive RRE means ISTRUM outperformed the compared method while 
the negative RRE means the compared method had higher accuracy than ISTRUM. The value of RRE indicates the percentage of improvement or degradation of ISTRUM when compared with STRUM, STDFA, ESTARFM and FSDAF.

\section{Results}

In this section, we present and analyze the experimental results. Sections 4.1-4.4 are based on results of experiment described in Section 3.2.1. Sections 4.5-4.7 are based on results of Sections 3.2.2-3.2.4, respectively.

\subsection{Accuracy Comparison of Derived Fine-Resolution Temporal Change Images}

Deriving accurate $\Delta \mathbf{F}$ is the key step for both ISTRUM and STRUM. The derived $\Delta \mathbf{F}$ of band 4 (B4) in experiment DOY187by123 was used to analyze the improvements of ISTRUM, because B4 is the near infrared (NIR) band which could well indicate the large temporal change from bare soil to vegetation occurred during DOY123 and DOY187. For DOY187by123, when $w_{h}$ was set to 1 for ISTRUM and STRUM, and $n_{m}$ was set to 3 for STRUM, both ISTRUM and STRUM had highest accuracy (according to Section 4.3). Therefore, the predicted Landsat-like images with the parameter settings were used in the analysis.

The $\Delta \mathbf{F}$ of B4 derived in ISTRUM and STRUM were shown in Figure 3. The $\Delta \mathbf{F}$ derived in ISTRUM pointed out more spectral variability and spatial details than the one derived in STRUM. Because, in ISTRUM, $\Delta \mathbf{F}$ is calculated by mixing the spectra of endmembers and the abundance image $\mathbf{A}_{F}^{B}$ which provides well representation of land surface, the mixture could generate different pixel values for $\Delta \mathbf{F}$ and reserve the spatial characteristics of $\mathbf{A}_{F}^{B}$, therefore, spectral variability and spatial details are well presented on $\Delta \mathbf{F}$. However, $\Delta \mathbf{F}$ is obtained by directly assigning spectra of endmembers to a hard-classification image in STRUM. Therefore, $\Delta \mathbf{F}$ derived in STRUM shows block effect because the footprints of MODIS pixels are obvious and fine pixels belong to same class type have identical values. Figure 4 shows the scatter plot of derived $\Delta \mathbf{F}$ versus actual temporal change of B4. Result of ISTRUM was more correlated with the actual $\triangle F$ than STRUM, which indicated the improved accuracy of ISTRUM.
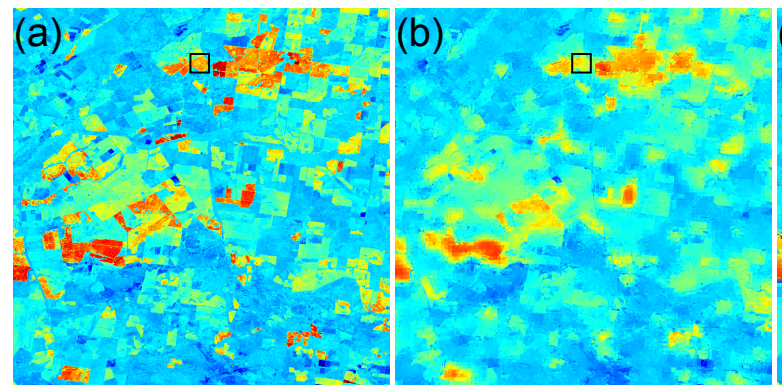

(d)

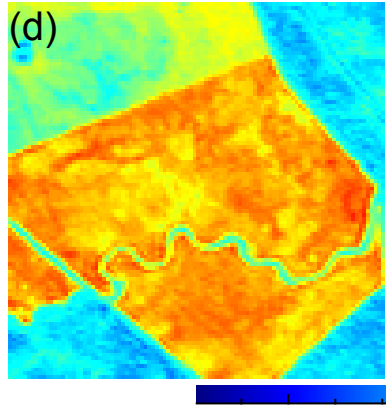

$-0.2$ (e)

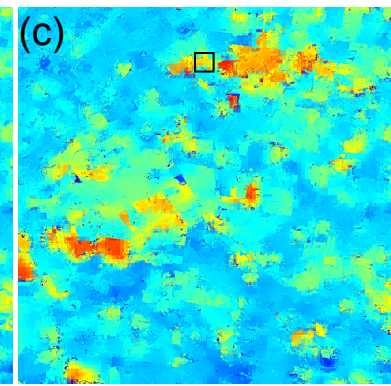

(f)

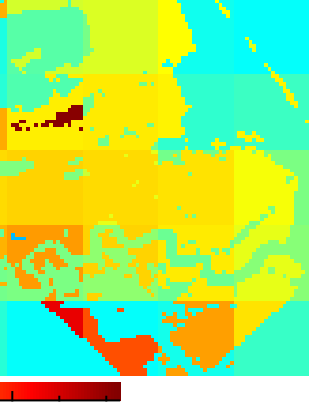

0.4

Figure 3. Comparison between actual and derived $\Delta \mathbf{F}$ of B4: (a) actual $\Delta \mathbf{F} ;(\mathbf{b}) \Delta \mathbf{F}$ derived by ISTRUM; and (c) $\Delta \mathbf{F}$ derived by STRUM. The $100 \times 100$ pixels in the black square of $(\mathbf{a}-\mathbf{c})$ are enlarged and shown in $(\mathbf{d}-\mathbf{f})$, respectively. 

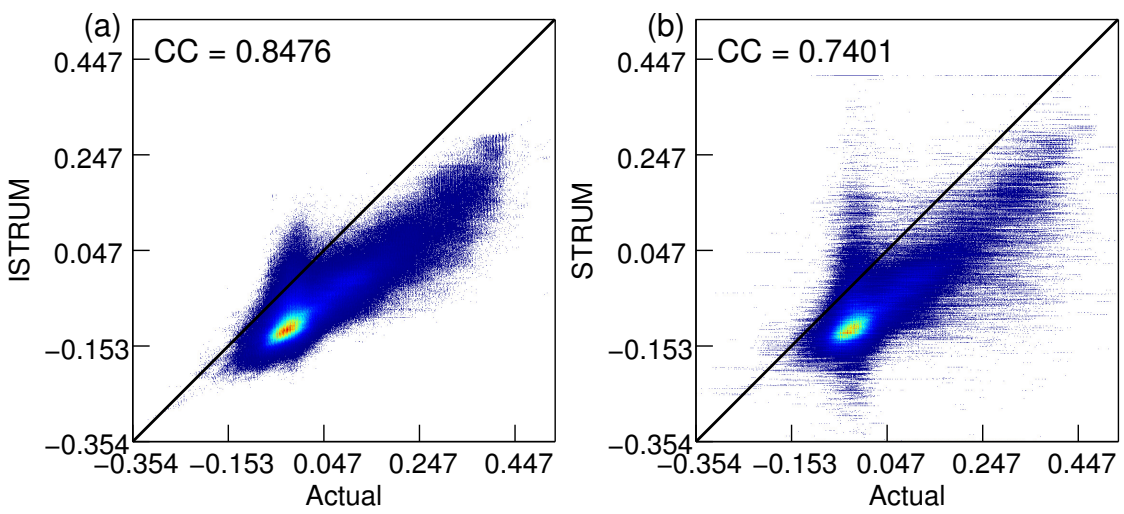

Figure 4. Scatter plot of actual $\Delta \mathbf{F}$ versus derived $\Delta \mathbf{F}$ of B4: (a) ISTRUM result; and (b) STRUM result.

\subsection{Accuracy Improvements of Every Step in ISTRUM}

The accuracy improvements of ISTRUM over STRUM were analyzed step by step with experiment DOY187by123 (Table 1). Abundance image rather than classification image was applied in ISTRUM, and this improvement was denoted as Improvement-1. Based on Improvement-1, the average increase of CC was about 0.0540 and the average decrease of RRMSE was about $2.99 \%$. The SAM decreased 1.022 and the $Q 2^{n}$ increased 0.049. Sensor difference was also considered in ISTRUM. The sensor difference adjustment (Improvement-2) increased CC about 0.0331 while decreased the RRMSE about $0.56 \%$. The SAM decreased 0.134 and the $Q 2^{n}$ increased 0.005 after sensor difference adjustment. According to RRE, the average accuracy improvement of ISTRUM is $23.15 \%$ in terms of CC and $12.62 \%$ of RRMSE.

Among all the bands (i.e., B1-B5 and B7), improvement of B4 is biggest for both CC and RRMSE values. Land surface changed from bare soil to vegetation between DOY123 and DOY187. The growth of vegetation resulted in large reflectance change of B4. Even though pixels in different regions belong to the same vegetation type, their reflectance change is different according to Figure 2. Deriving accurate $\Delta \mathbf{F}$ of $B 4$ that takes intra-class spectral variability into account will better improve the accuracy. ISTRUM is capable to derive $\Delta \mathbf{F}$ with spectral variability and spatial details. Therefore, it generated biggest improvement in B4.

Table 1. Accuracy improvements of every step in ISTRUM. Improvement-1 denotes the change of accuracy based on STRUM when abundance image is applied. Improvement-2 denotes the change of accuracy based on Improvement-1 when sensor difference is adjusted. Accuracy of ISTRUM equals to accuracy of STRUM adds Improvement- 1 and Improvement-2.

\begin{tabular}{ccccccccc}
\hline & & B1 & B2 & B3 & B4 & B5 & B7 & Mean \\
\hline \multirow{6}{*}{ CC } & STRUM & 0.5589 & 0.6283 & 0.6492 & 0.6477 & 0.6735 & 0.5700 & 0.6213 \\
& Improvement-1 & +0.0384 & +0.0249 & +0.0497 & +0.1066 & +0.0458 & +0.0587 & +0.0540 \\
& Improvement-2 & +0.0686 & +0.0321 & +0.0437 & +0.0040 & +0.0268 & +0.0234 & +0.0331 \\
& ISTRUM & 0.6658 & 0.6853 & 0.7425 & 0.7583 & 0.7461 & 0.6521 & 0.7084 \\
& Overall & +0.1070 & +0.0570 & +0.0934 & $\mathbf{+ 0 . 1 1 0 6}$ & +0.0726 & +0.0821 & +0.0871 \\
& RRE (\%) & 24.25 & 15.33 & 26.61 & 31.40 & 22.24 & 19.09 & 23.15 \\
\hline \multirow{4}{*}{ RRMSE } & STRUM & 31.1155 & 24.4656 & 32.5806 & 29.9281 & 18.3441 & 32.9287 & 28.2271 \\
$(\%)$ & Improvement-1 & -2.2637 & -1.4984 & -3.0815 & -5.6265 & -1.8112 & -3.6469 & -2.9880 \\
& Improvement-2 & +1.6177 & -1.3239 & -1.9427 & -0.1755 & -0.7202 & -0.7985 & -0.5572 \\
& ISTRUM & 30.4694 & 21.6433 & 27.5564 & 24.1260 & 15.8128 & 28.4833 & 24.6819 \\
& Overall & -0.6460 & -2.8223 & -5.0241 & -5.8020 & -2.5313 & -4.4453 & -3.5452 \\
& RRE (\%) & 2.08 & 11.54 & 15.42 & 19.39 & 13.80 & 13.50 & 12.62 \\
\hline
\end{tabular}




\subsection{Accuracy ISTRUM and STRUM with Different User Defined Parameters}

The user defined parameters $n_{m}$ and $w_{h}$ could influence the accuracy of synthetic images [26]. We first compared the performance of ISTRUM and STRUM with the experiments of DOY123by107, DOY187by123 and DOY235by187. $n_{m}$ was set to 3, 5, 7, and 9 for STRUM and $w_{h}$ was set to 2 . The result (Figure 5) indicates ISTRUM always outperformed STRUM. The accuracy of STRUM decreased as $n_{m}$ increased. The conclusion was also drawn by Gevaert and García-Haro [26] because the number of unknowns in spatial-unmixing increases when $n_{m}$ is large. The result implies SVD model is suitable to integrate into ISTRUM because it has three endmembers $\left(n_{m}=3\right)$.
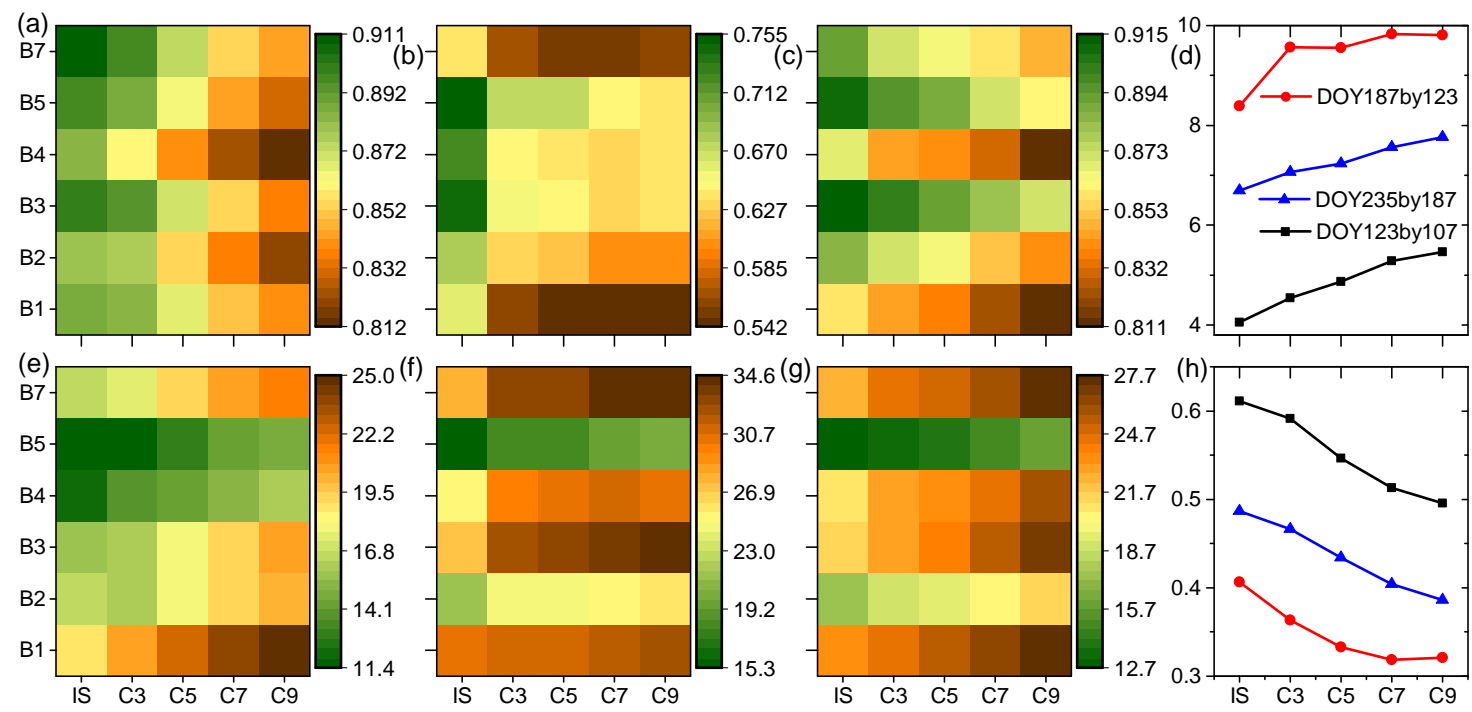

Figure 5. Accuracy of ISTRUM and STRUM with different $n_{m}$ : (a,e) heat maps, where the greener is the block, the higher is the accuracy, for CC and RRMSE (\%) for experiments DOY123by107; (b,f) the same as (a,e) for DOY187by123; and (c,g) the same as (a,e) for DOY235by187; (d) SAM; and (h) Q2 ${ }^{n}$. "IS" denotes ISTRUM and "C3", "C5", “C7", and "C9" denote STRUM with $n_{m}$ equal to 3, 5, 7, and 9, respectively.

To assess the influence of sliding-window size, $w_{h}$ was set to $\{1,2, \ldots, 10\}$ (i.e., $w \in\{3,5, \ldots, 21\}$ ) and performance of ISTRUM and STRUM was compared. $n_{m}$ of STRUM was set to 3 in the experiment. As $w$ increased, accuracy of both ISTRUM and STRUM decreased (Figure 6). $w$ determines the number of equations to be solved in the spatial-unmixing process. A large $w$ could induce uncertainties in calculation of $\Delta \mathbf{E}_{C}$ because there are many equations in the computation. In addition, large $w$ means reflectance change of same endmember should be same in a large region. The assumption may be violated because the reflectance change can be spatially varied. Hence, it could induce errors in solving $\Delta \mathbf{E}_{C}$. Optimal $w$ can be determined with methods proposed in [48]. The optimal $w_{h}$ is 2 for DOY123by107 and 1 for DOY187by123 and DOY235by187 in the study area. $w_{h}$ was set to 1 in the following experiments for convenience. The result also revealed ISTRUM outperforms STRUM nearly for every band and sliding-window size. Because based on the improvements which include the utilization of abundance image and the adjustment of sensor difference, ISTRUM could derive more accurate $\Delta \mathbf{F}$ than STRUM. 

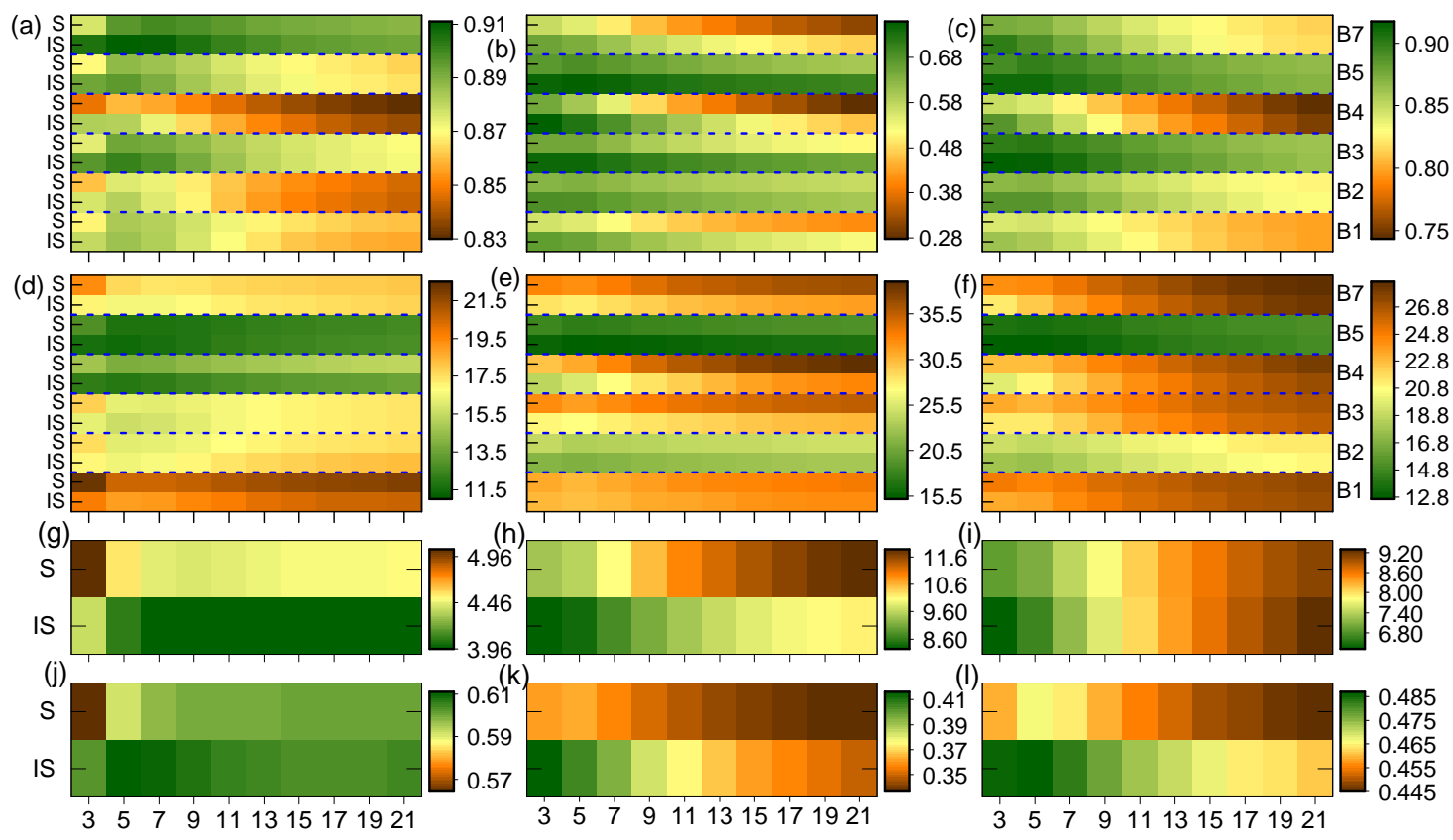

$\begin{array}{llllllllll}3 & 5 & 7 & 9 & 11 & 13 & 15171921\end{array}$

Figure 6. Accuracy of ISTRUM and STRUM with different $w:(\mathbf{a}, \mathbf{d}, \mathbf{g}, \mathbf{j})$ heat maps for CC, RRMSE (\%), SAM and $Q 2^{n}$ for experiments DOY123by107; (b,e,h,k) the same as $(\mathbf{a}, \mathbf{d}, \mathbf{g}, \mathbf{j})$ for DOY187by123; and $(\mathbf{c}, \mathbf{f}, \mathbf{i}, \mathbf{l})$ the same as $(\mathbf{a}, \mathbf{d}, \mathbf{g}, \mathbf{j})$ for DOY235by187. The numbers of X-axis denote the value of $w$. The "IS" and "S" on left Y-axis denote ISTRUM and STRUM method, respectively. The blue dash lines in (a-f) divide the accuracy of ISTRUM and STRUM for each band of the image.

\subsection{Influence of Base L-C Image Pair on ISTRUM}

ISTRUM synthesizes Landsat-like image $\mathbf{F}^{P \_}{ }^{B}$ by adding $\Delta \mathbf{F}$ to $\mathbf{F}^{B}$ (Equation (13)). Accuracy of $\mathbf{F}^{P}{ }^{B}$ is influenced by the selected base Landsat image $\mathbf{F}^{B}$ and the derived $\Delta \mathbf{F}$. Once the input L-C image pair is selected in the prediction, $\mathbf{F}^{B}$ is determined. We denote the $C C$ between $\mathbf{F}^{B}$ and $\mathbf{F}^{P}$ as inherent $C$ (ICC). We also denote the $C C$ between $\mathbf{F}^{P}$ and $\mathbf{F}^{P-B}$, which is a metric of the accuracy of $\mathbf{F}^{P_{-} B}$, as predicted CC (PCC). High ICC can help to generate high PCC to some degree. To analyze influence of base L-C image pair, ICC was calculated and shown in Table 2. In addition, CC between $\mathbf{C}^{B}$ and $\mathbf{C}^{P}$ was also calculated and denoted as $\mathrm{CC}$ of coarse-resolution images (CCC).

Table 2. ICC, CCC and PCC of experiment combinations DOY123by107, DOY123by107 and DOY235by187.

\begin{tabular}{cccccccccc}
\hline & \multicolumn{3}{c}{ DOY123by107 } & \multicolumn{3}{c}{ DOY187by123 } & \multicolumn{3}{c}{ DOY235by187 } \\
\cline { 2 - 10 } & ICC & CCC & PCC & ICC & CCC & PCC & ICC & CCC & PCC \\
\hline B1 & 0.8563 & 0.8029 & $\mathbf{0 . 8 7 9 6}$ & 0.2244 & 0.0647 & $\mathbf{0 . 6 6 5 8}$ & 0.7686 & 0.7937 & $\mathbf{0 . 8 6 3 3}$ \\
B2 & 0.8350 & 0.7678 & $\mathbf{0 . 8 7 7 2}$ & 0.4097 & 0.4212 & $\mathbf{0 . 6 8 5 3}$ & 0.7862 & 0.7111 & $\mathbf{0 . 8 8 6 8}$ \\
B3 & 0.8621 & 0.8106 & $\mathbf{0 . 8 9 6 8}$ & 0.1791 & 0.0886 & $\mathbf{0 . 7 4 2 5}$ & 0.8422 & 0.8125 & $\mathbf{0 . 9 1 7 2}$ \\
B4 & 0.8211 & 0.7916 & $\mathbf{0 . 8 8 3 0}$ & -0.1429 & -0.2370 & $\mathbf{0 . 7 5 8 3}$ & 0.6592 & 0.6380 & $\mathbf{0 . 8 8 5 7}$ \\
B5 & 0.8632 & 0.7647 & $\mathbf{0 . 8 9 2 7}$ & 0.3662 & 0.2403 & $\mathbf{0 . 7 4 6 1}$ & 0.8387 & 0.8439 & $\mathbf{0 . 9 1 0 5}$ \\
B7 & 0.8944 & 0.8206 & $\mathbf{0 . 9 0 6 3}$ & 0.0933 & -0.0754 & $\mathbf{0 . 6 5 2 1}$ & 0.7833 & 0.7628 & $\mathbf{0 . 9 0 0 9}$ \\
Mean & 0.8554 & 0.7930 & $\mathbf{0 . 8 8 9 3}$ & 0.1883 & 0.0837 & $\mathbf{0 . 7 0 8 4}$ & 0.7797 & 0.7603 & $\mathbf{0 . 8 9 4 1}$ \\
\hline
\end{tabular}

Accuracy of DOY123by107 and DOY235by187 were much higher than DOY187by123, which might be partially attributed to their high ICC values. The result also indicated that if the L-C pair with high ICC is available, the user should select it in the application of ISTRUM. However, $\mathbf{F}^{P}$ is not 
observed in actual application and ICC cannot be calculated in advance. Since CCC has similar trends with ICC, it could be computed in advance and guide the selection of L-C pair in the application.

For DOY187by123, land cover changed from bare soil to vegetation in large regions between DOY123 and DOY187. It caused the low ICC and violated the assumption of ISTRUM that abundance image should be same between $T_{B}$ and $T_{P}$. However, PCC is much higher than ICC which indicates the derived $\Delta \mathbf{F}$ in ISTRUM contributed a lot to the prediction of the Landsat-like image. Although the overall accuracy of synthesized Landsat-like image is not high in DOY187by123, the results implied ISTRUM does not heavily rely on the assumption that abundance image should be same between $T_{B}$ and $T_{P}$, and is capable to capture temporal change when the land cover type changed.

\subsection{Influence of Endmember Variability on ISTRUM}

\subsubsection{Sensitivity of ISTRUM to the Endmember Variability}

The variation in endmembers' spectra results in uncertainty of the estimated $\mathbf{A}_{F}^{B}$ in spectralunmixing which may further affect the accuracy of ISTRUM. To analyze the sensitivity of ISTRUM to the endmember variability, Landsat image on DOY187 was unmixed with different spectra combinations of SVD, and estimated abundance images were applied to synthesize Landsat-like image on DOY235. Since the approach to extract endmembers selects a suite of pure pixels for $\mathrm{S}, \mathrm{V}$, and $\mathrm{D}$, the mean and standard deviation values (denoted as $\mu$ and $\sigma$ ) of the pixels were used to represent the range of endmember variability.

Considering the $\mu$ and $\sigma$ values of SVD spectra (Figure 7) extracted from Landsat image on DOY187, $\left\{\mathrm{S}_{\mu-4 \sigma}, \mathrm{S}_{\mu}, \mathrm{S}_{\mu+4 \sigma}\right\},\left\{\mathrm{V}_{\mu-6 \sigma}, \mathrm{V}_{\mu}, \mathrm{V}_{\mu+6 \sigma}\right\}$, and $\left\{\mathrm{D}_{\mu-\sigma}, \mathrm{D}_{\mu}, \mathrm{D}_{\mu+\sigma}\right\}$ were used to represent the variability of SVD. There were totally $27(3 \times 3 \times 3)$ spectra combinations of SVD (Table 3). Landsat image on DOY187 was unmixed by each combination. Absolute differences between abundance images unmixed by ID 1 26 and ID 0 were calculated and summarized in Figure 8. Variation of endmember spectra leads to obvious changes of abundance especially for $\mathrm{S}$ and $\mathrm{D}$. The maximum absolute abundance change is 0.1883 for $S$ and 0.1922 for D with spectra combination ID 14. Even though the variation of spectral is $6 \sigma$ for $\mathrm{V}$, the relative variation is small and the maximum absolute abundance change is 0.0765 for $\mathrm{V}$ with spectra combination ID 10.
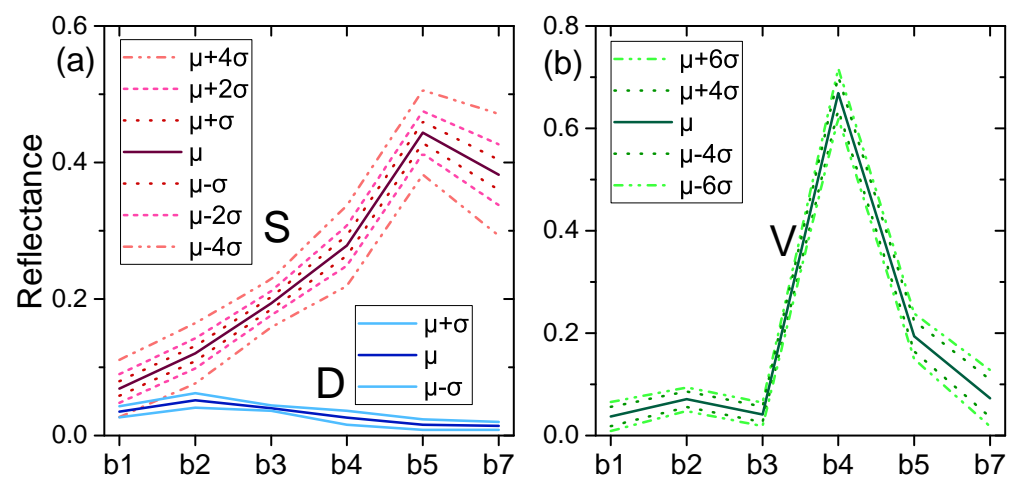

Figure 7. Mean and standard deviation of manually extracted SVD spectra in Landsat image on DOY187: (a) for S and D; and (b) for V. 
Table 3. The ID of each SVD spectra combination.

\begin{tabular}{cccccccccccc}
\hline ID & $\mathbf{S}$ & $\mathbf{V}$ & $\mathbf{D}$ & $\mathbf{I D}$ & $\mathbf{S}$ & $\mathbf{V}$ & $\mathbf{D}$ & $\mathbf{I D}$ & $\mathbf{S}$ & $\mathbf{V}$ & $\mathbf{D}$ \\
\hline 0 & $03 \mathrm{~S}_{\mu}$ & $\mathrm{V}_{\mu}$ & $\mathrm{D}_{\mu}$ & 9 & $\mathrm{~S}_{\mu+4 \sigma}$ & $\mathrm{V}_{\mu}$ & $\mathrm{D}_{\mu}$ & 18 & $\mathrm{~S}_{\mu-4 \sigma}$ & $\mathrm{V}_{\mu}$ & $\mathrm{D}_{\mu}$ \\
1 & $\mathrm{~S}_{\mu+4 \sigma}$ & $\mathrm{V}_{\mu+6 \sigma}$ & $\mathrm{D}_{\mu+\sigma}$ & 10 & $\mathrm{~S}_{\mu-4 \sigma}$ & $\mathrm{V}_{\mu+6 \sigma}$ & $\mathrm{D}_{\mu+\sigma}$ & 19 & $\mathrm{~S}_{\mu}$ & $\mathrm{V}_{\mu+6 \sigma}$ & $\mathrm{D}_{\mu+\sigma}$ \\
2 & $\mathrm{~S}_{\mu+4 \sigma}$ & $\mathrm{V}_{\mu+6 \sigma}$ & $\mathrm{D}_{\mu-\sigma}$ & 11 & $\mathrm{~S}_{\mu-4 \sigma}$ & $\mathrm{V}_{\mu+6 \sigma}$ & $\mathrm{D}_{\mu-\sigma}$ & 20 & $\mathrm{~S}_{\mu}$ & $\mathrm{V}_{\mu+6 \sigma}$ & $\mathrm{D}_{\mu-\sigma}$ \\
3 & $\mathrm{~S}_{\mu+4 \sigma}$ & $\mathrm{V}_{\mu+6 \sigma}$ & $\mathrm{D}_{\mu}$ & 12 & $\mathrm{~S}_{\mu-4 \sigma}$ & $\mathrm{V}_{\mu+6 \sigma}$ & $\mathrm{D}_{\mu}$ & 21 & $\mathrm{~S}_{\mu}$ & $\mathrm{V}_{\mu+6 \sigma}$ & $\mathrm{D}_{\mu}$ \\
4 & $\mathrm{~S}_{\mu+4 \sigma}$ & $\mathrm{V}_{\mu-6 \sigma}$ & $\mathrm{D}_{\mu+\sigma}$ & 13 & $\mathrm{~S}_{\mu-4 \sigma}$ & $\mathrm{V}_{\mu-6 \sigma}$ & $\mathrm{D}_{\mu+\sigma}$ & 22 & $\mathrm{~S}_{\mu}$ & $\mathrm{V}_{\mu-6 \sigma}$ & $\mathrm{D}_{\mu+\sigma}$ \\
5 & $\mathrm{~S}_{\mu+4 \sigma}$ & $\mathrm{V}_{\mu-6 \sigma}$ & $\mathrm{D}_{\mu-\sigma}$ & 14 & $\mathrm{~S}_{\mu-4 \sigma}$ & $\mathrm{V}_{\mu-6 \sigma}$ & $\mathrm{D}_{\mu-\sigma}$ & 23 & $\mathrm{~S}_{\mu}$ & $\mathrm{V}_{\mu-6 \sigma}$ & $\mathrm{D}_{\mu-\sigma}$ \\
6 & $\mathrm{~S}_{\mu+4 \sigma}$ & $\mathrm{V}_{\mu-6 \sigma}$ & $\mathrm{D}_{\mu}$ & 15 & $\mathrm{~S}_{\mu-4 \sigma}$ & $\mathrm{V}_{\mu-6 \sigma}$ & $\mathrm{D}_{\mu}$ & 24 & $\mathrm{~S}_{\mu}$ & $\mathrm{V}_{\mu-6 \sigma}$ & $\mathrm{D}_{\mu}$ \\
7 & $\mathrm{~S}_{\mu+4 \sigma}$ & $\mathrm{V}_{\mu}$ & $\mathrm{D}_{\mu+\sigma}$ & 16 & $\mathrm{~S}_{\mu-4 \sigma}$ & $\mathrm{V}_{\mu}$ & $\mathrm{D}_{\mu+\sigma}$ & 25 & $\mathrm{~S}_{\mu}$ & $\mathrm{V}_{\mu}$ & $\mathrm{D}_{\mu+\sigma}$ \\
8 & $\mathrm{~S}_{\mu+4 \sigma}$ & $\mathrm{V}_{\mu}$ & $\mathrm{D}_{\mu-\sigma}$ & 17 & $\mathrm{~S}_{\mu-4 \sigma}$ & $\mathrm{V}_{\mu}$ & $\mathrm{D}_{\mu-\sigma}$ & 26 & $\mathrm{~S}_{\mu}$ & $\mathrm{V}_{\mu}$ & $\mathrm{D}_{\mu-\sigma}$ \\
\hline
\end{tabular}

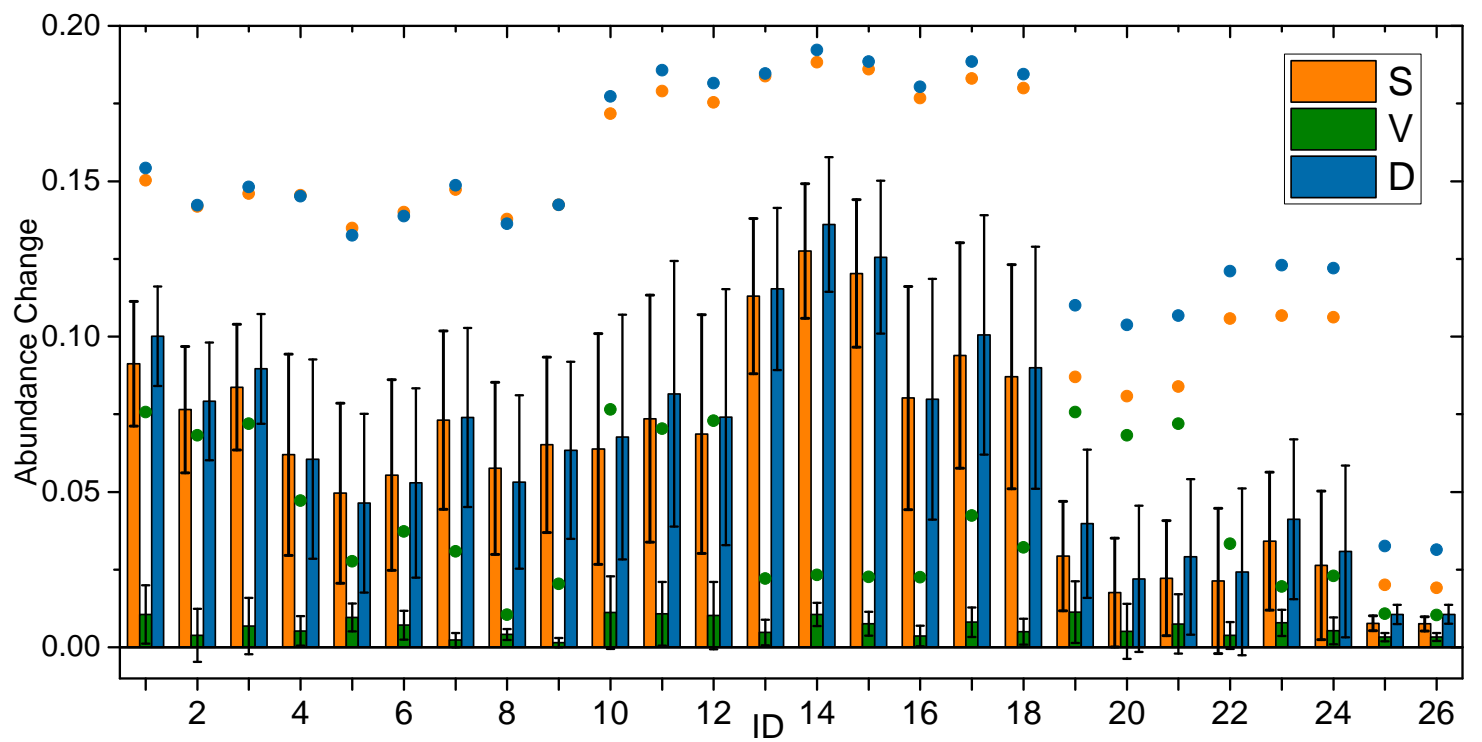

Figure 8. Absolute differences of abundance unmixed by ID $1 \sim 26$ from ID 0 . The filled points denote max value, the bars denote mean value and the whiskers denote standard deviation of the absolute abundance changes.

Each abundance image was separately applied in the following steps of ISTRUM and a total of 27 Landsat-like images on DOY257 were synthesized. Accuracy of each image was calculated and shown in Figure 9. It can be seen that variations of CC, RRMSE, SAM and $Q 2^{n}$ are small, even though endmember variability resulted in large variation of the abundance images. Maximum (max) and minimum ( $\mathrm{min}$ ) values of CC, RRMSE, SAM and $Q 2^{n}$ were identified. Table 4 shows the maximum and minimum values of CC and RRMSE. Average dynamics for CC and RRMSE are $0.0033 \%$ and $0.3446 \%$, respectively. Dynamic range is 6.2837-6.3782 for SAM and 0.4834-0.4888 for $Q 2^{n}$. The variation of accuracy is small, even though different spectra of endmembers were applied in spectral-unmixing process of ISTRUM. The results indicated ISTRUM was robust to the endmember variability.

Table 4. Dynamic ranges of CC and RRMSE for each band (B1-B5 and B7) of the synthesized Landsatlike images.

\begin{tabular}{|c|c|c|c|c|c|c|c|c|}
\hline & & B1 & B2 & B3 & B4 & B5 & B7 & Mean \\
\hline \multirow[t]{2}{*}{$\mathrm{CC}$} & $\min$ & 0.8619 & 0.8854 & 0.9157 & 0.8841 & 0.9099 & 0.8996 & 0.8928 \\
\hline & $\max$ & 0.8654 & 0.8890 & 0.9194 & 0.8879 & 0.9118 & 0.9028 & 0.8961 \\
\hline \multirow{2}{*}{$\begin{array}{c}\text { RRMSE } \\
(\%)\end{array}$} & $\min$ & 23.1530 & 17.5253 & 20.9421 & 19.6332 & 12.5850 & 21.1355 & 19.1623 \\
\hline & $\max$ & 23.4393 & 17.8413 & 21.4912 & 20.0087 & 12.6957 & 21.5655 & 19.5070 \\
\hline
\end{tabular}



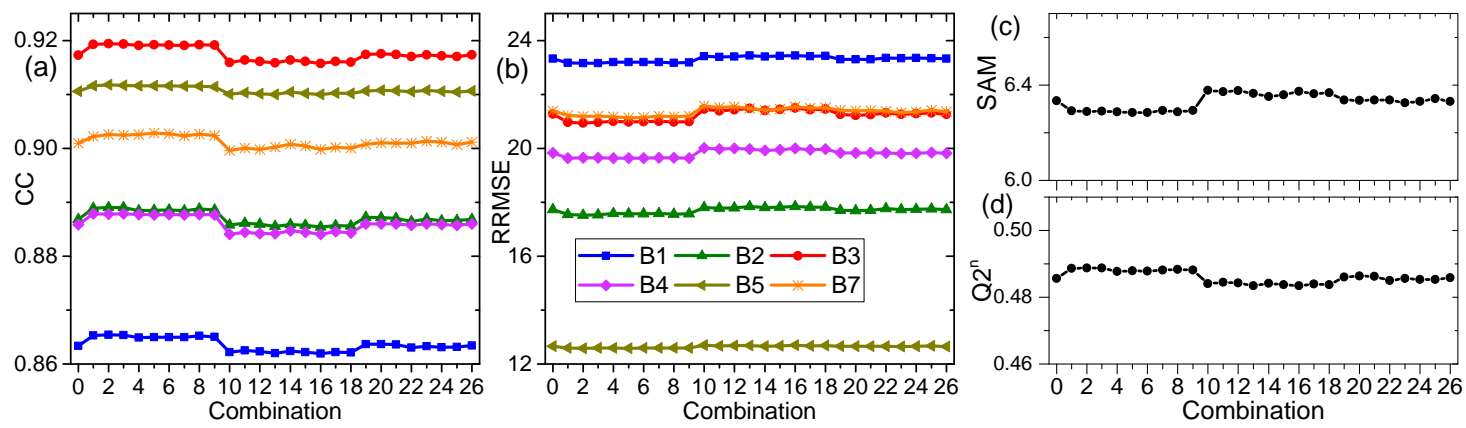

Figure 9. Accuracy of predicted Landsat-like images on DOY 257 with different spectra combinations of SVD: (a) CC; (b) RRMSE (\%); (c) SAM; and (d) $Q 2^{n}$.

The endmember variability in spectral-unmixing leads to the variation of $\mathbf{A}_{F}^{B}$. Therefore, the aggregated $\mathbf{A}_{C}^{B}$ also varied. Because $\mathbf{A}_{C}^{B}$ was directly applied in spatial-unmixing, the derived $\Delta \mathbf{E}_{C}$ and $\Delta \mathbf{E}_{F}$ were also varied. However, $\Delta \mathbf{E}_{F}$ was linear mixed with $\mathbf{A}_{F}^{B}$, and the uncertainties in $\Delta \mathbf{E}_{F}$ and $\mathbf{A}_{F}^{B}$ may be counteracted in the derived $\Delta \mathbf{F}$, which directly affects the accuracy of synthetic Landsat-like image.

The residual of spatial-umixing in each experiment were also calculated for further analysis. For coarse pixel $\left[i_{c}, j_{c}, b\right]$, derived $\Delta \mathbf{E}_{C}[*, b]$ was mixed with $\mathbf{A}_{C}^{B}\left[i_{c}, j_{c}, *\right]$. The residual $\mathcal{E}\left[i_{c}, j_{c}, b\right]$ is the difference between $\Delta \mathbf{C}\left[i_{c}, j_{c}, b\right]$ and the mixture value. The mean absolute value of residual barely changed for each spectra combination of SVD (Figure 10), which indicated the mixture of $\Delta \mathbf{E}_{C}$ and $\mathbf{A}_{C}^{B}$ changed little. Since $\mathbf{A}_{C}^{B}$ is aggregated from $\mathbf{A}_{F}^{B}$, and $\Delta \mathbf{E}_{F}$ is linearly adjusted based on $\Delta \mathbf{E}_{C}$. The $\Delta \mathbf{F}$, which is mixture of $\Delta \mathbf{E}_{F}$ and $\mathbf{A}_{F}^{B}$, should also be stable and guaranteed the stability of synthetic Landsat-like image.
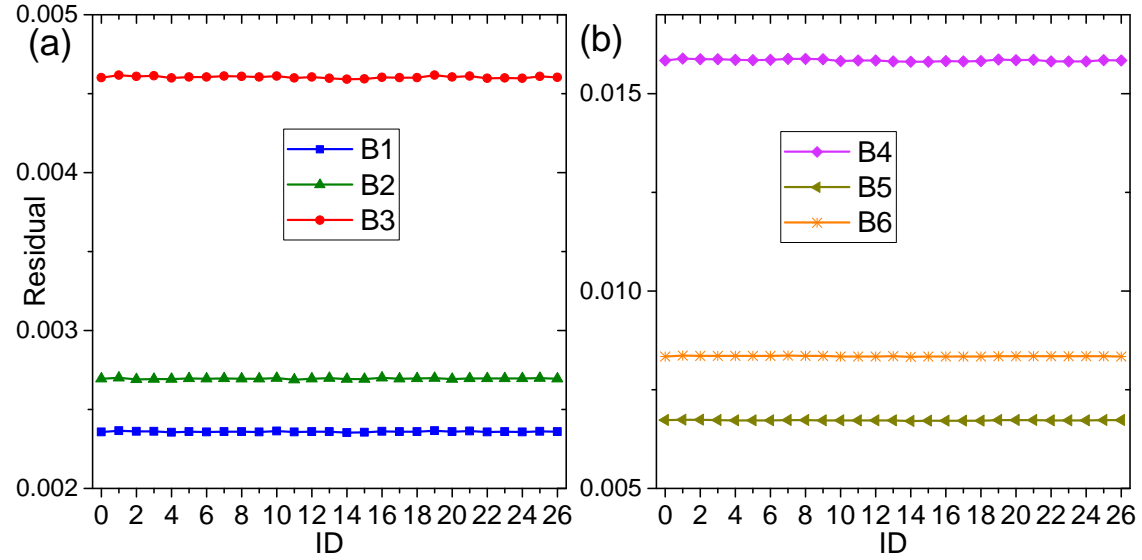

Figure 10. Mean absolute residual in the spatial-umixing of $\Delta \mathrm{C}$ for each spectra combination of SVD.

\subsubsection{Applicability of Global SVD Endmembers}

As ISTRUM was not sensitive to the endmember variability, applicability of Global SVD endmembers [49] was tested in this experiment. Landsat image on DOY107, DOY123 and DOY187 were unmixed by the Global SVD, respectively, and Landsat-like images on their following date were synthesized. The accuracy were compared with experiments when endmembers were extracted from the image itself. Figure 11 shows the differences between Global SVD and extracted SVD spectra. Although the spectra were obviously different, the accuracy varied little and even was improved when Global SVD (Table 5) was used. The results implied the potential to directly apply Global SVD endmembers in future applications of ISTRUM. Since SVD is a representative spectral mixture model for globally archived Landsat TM/ETM+/OLI images and the Global SVD endmembers are extracted 
from a global Landsat dataset $[39,49,50]$. The integration of SVD model strengthens the applicability of ISTRUM for Landsat images observed on a global scale. The direct utilization of the Global SVD endmembers makes ISTRUM friendly to use because only $w_{h}$ needs to be defined by the users.

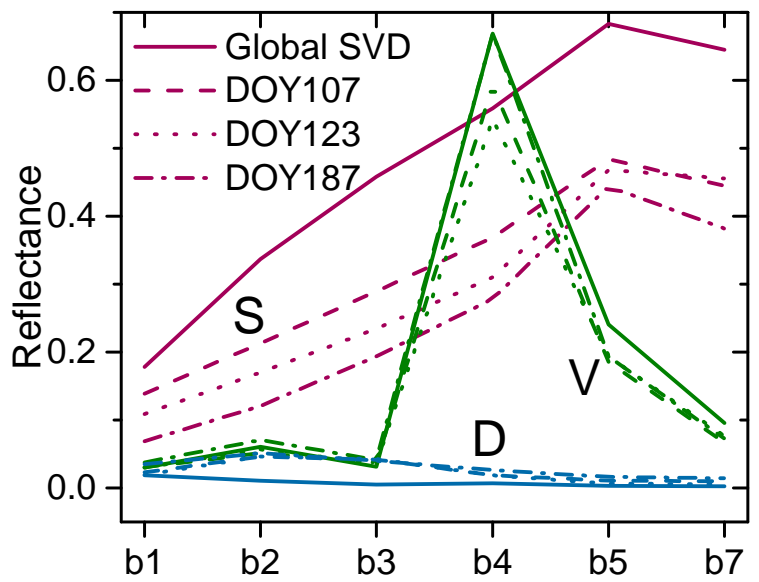

Figure 11. Comparison between Global SVD and manually extracted SVD spectra.

Table 5. Accuracy of ISTRUM when manually extracted SVD (SVD1) and Global SVD (SVD2) were applied separately.

\begin{tabular}{|c|c|c|c|c|c|c|c|}
\hline & & \multicolumn{2}{|c|}{ DOY123by107 } & \multicolumn{2}{|c|}{ DOY187by123 } & \multicolumn{2}{|c|}{ DOY235by187 } \\
\hline & & SVD1 & SVD2 & SVD1 & SVD2 & SVD1 & SVD2 \\
\hline \multirow{7}{*}{$\mathrm{CC}$} & B1 & 0.8796 & 0.8850 & 0.6658 & 0.6647 & 0.8633 & 0.8692 \\
\hline & $\mathrm{B} 2$ & 0.8772 & 0.8821 & 0.6853 & 0.6828 & 0.8868 & 0.8943 \\
\hline & B3 & 0.8968 & 0.9010 & 0.7425 & 0.7369 & 0.9172 & 0.9238 \\
\hline & B4 & 0.8830 & 0.8841 & 0.7583 & 0.7460 & 0.8857 & 0.8898 \\
\hline & B5 & 0.8927 & 0.8963 & 0.7461 & 0.7433 & 0.9105 & 0.9146 \\
\hline & B7 & 0.9063 & 0.9098 & 0.6521 & 0.6413 & 0.9009 & 0.9054 \\
\hline & Mean & 0.8893 & 0.8930 & 0.7084 & 0.7025 & 0.8941 & 0.8995 \\
\hline \multirow{7}{*}{ RRMSE (\%) } & B1 & 19.7897 & 19.3336 & 30.4694 & 30.3104 & 23.3271 & 22.8452 \\
\hline & $\mathrm{B} 2$ & 16.9933 & 16.6672 & 21.6433 & 21.7683 & 17.7299 & 17.0448 \\
\hline & B3 & 16.2304 & 15.9122 & 27.5564 & 27.7627 & 21.2792 & 20.2884 \\
\hline & B4 & 12.1429 & 12.0233 & 24.1260 & 24.6530 & 19.8399 & 19.4376 \\
\hline & B5 & 11.6851 & 11.4985 & 15.8128 & 15.9536 & 12.6622 & 12.3848 \\
\hline & B7 & 17.0202 & 16.7432 & 28.4833 & 28.8972 & 21.3935 & 20.7886 \\
\hline & Mean & 15.6436 & 15.3630 & 24.6819 & 24.8909 & 19.3719 & 18.7983 \\
\hline SAM & - & 4.3867 & 4.2934 & 8.2561 & 8.3265 & 6.3366 & 6.1858 \\
\hline$Q 2^{n}$ & - & 0.6025 & 0.6119 & 0.4145 & 0.4179 & 0.4855 & 0.4947 \\
\hline
\end{tabular}

\subsection{Experiment with Two L-C Pairs}

To test ISTRUM with more than one L-C pairs, Landsat-like images on DOY123 and DOY187 were predicted by L-C pairs on DOY107 and DOY235. The Global SVD endmembers were used in the spectral-unmixing and $w_{h}$ was set to 1 . The results are shown in Figure 12 . The prediction accuracy was low when there was one L-C pair and significant temporal change occurred between $T_{B}$ and $T_{P}$. However, the accuracy was improved when another L-C pair with small temporal change was applied. The accuracy of Landsat-like image which was synthesized by two L-C pairs was higher than the one which was synthesized by either one of two L-C pairs. However, for synthetic image on DOY123 based on two L-C pairs, accuracy of B4, B5, and B7 decreased slightly when compared with the image synthesized by one L-C pair on DOY107. Because the long time interval between 
DOY123 and DOY235 (112 days) can complicate the temporal change of land surface, which degrades the accuracy of prediction if land cover type have changed.
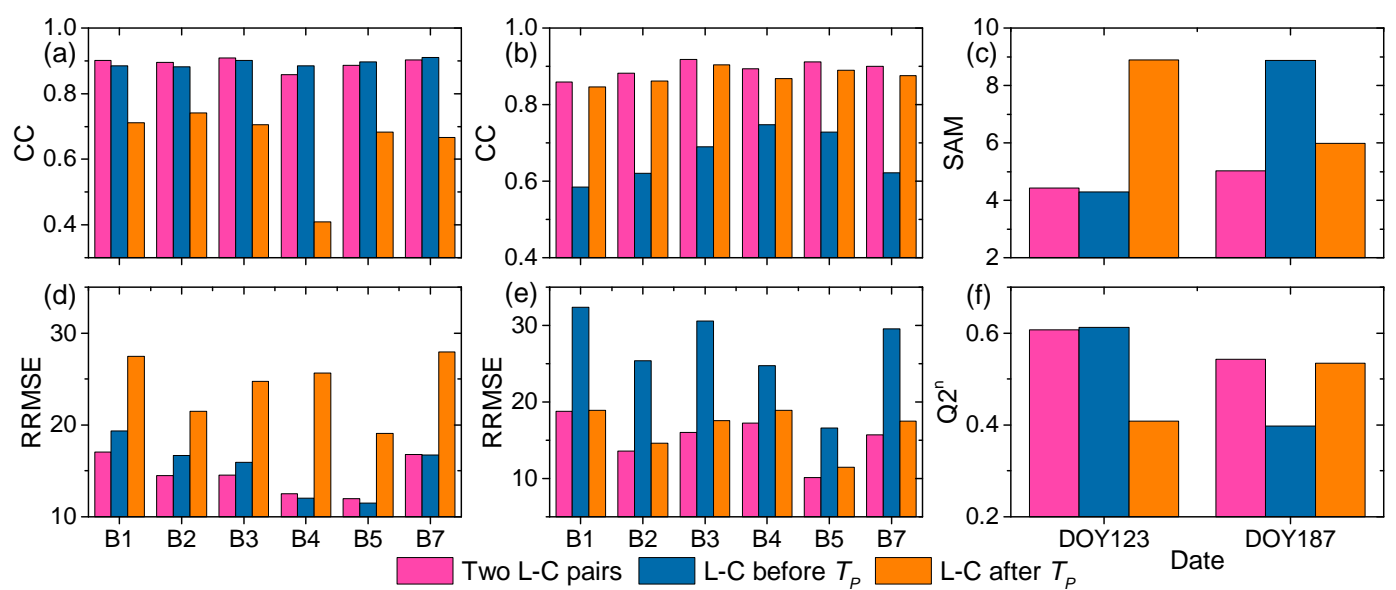

Figure 12. Accuracy comparison of ISTRUM with one and two L-C pairs: (a,d) CC and RRMSE (\%) for prediction result of DOY123; (b,e) the same as $(\mathbf{a}, \mathbf{d})$ for DOY187; and $(\mathbf{c}, \mathbf{f})$ SAM and $Q 2^{n}$.

\subsection{Comparisons of ISTRUM, STDFA, ESTARFM, HCM, and FSDAF}

ISTRUM was compared with STDFA, ESTARFM, HCM, and FSDAF, which are benchmark spatiotemporal image fusion methods. Performance of different methods was evaluated in terms of quantitative accuracy and computational efficiency.

\subsubsection{Quantitative Accuracy Comparison}

Table 6 shows the accuracy of STDFA, HCM, FSDAF and ISTRUM performed with one L-C pair.

It can be seen that ISTRUM always outperformed STDFA which is another spatial-unmixing based method. Average accuracy of ISTRUM is generally 10\% higher than STDFA. Classification image is applied in spatial-unmixing process of STDFA, thus, downscaled fine-resolution image lacks spectral variability. In addition, in order to apply the Surface Reflectance Calculation Model (SRCM), STDFA performs spatial-unmixing to coarse-resolution image on $T_{B}$ and $T_{P}$ separately [25]. The two spatial-unmixing processes can increase uncertainties and induce errors.

ISTRUM outperformed HCM slightly in DOY187by123 and DOY235by187, while HCM was slightly better in DOY123by107. The transformation matrix learned from coarse-resolution images heavily influences the accuracy of HCM. Time interval is 16 days for DOY123by107 and temporal change is insignificant. The strong correlation between images on DOY123 and DOY187 (Table 2) contributed to deriving a reliable transformation matrix in the learning process. Therefore, HCM had higher accuracy. However, significant temporal change occurred for DOY187by123 and DOY235by187 due to the long time interval. The change degraded the accuracy of transformation matrix in HCM. Thus, ISTRUM outperformed HCM. The result indicates ISTRUM has better performance than HCM when temporal change is significant. 
Table 6. Quantitative assessment of synthesized Landsat-like images by STDFA, HCM, FADAF and ISTRUM. The $\mathrm{R}$ in the second parentheses denotes the rank of the method.

\begin{tabular}{|c|c|c|c|c|c|c|}
\hline & & & STDFA(RRE \%)(R) & HCM(RRE \%)(R) & FSDAF(RRE \%)(R) & ISTRUM(R) \\
\hline \multirow{16}{*}{$\begin{array}{c}\text { DOY123 } \\
\text { by107 }\end{array}$} & \multirow{7}{*}{$\mathrm{CC}$} & B1 & $0.8660(14.17)(4)$ & $0.8949(-9.41)(2)$ & $0.8970(-11.66)(1)$ & $0.8850(3)$ \\
\hline & & B2 & $0.8556(18.32)(4)$ & $0.8939(-11.20)(2)$ & $0.8951(-12.43)(1)$ & $0.8821(3)$ \\
\hline & & B3 & $0.8710(23.24)(4)$ & $0.9045(-3.70)(2)$ & $0.9095(-9.35)(1)$ & $0.9010(3)$ \\
\hline & & B4 & $0.8424(26.49)(4)$ & $0.8820(1.79)(3)$ & $0.8896(-4.96)(1)$ & $0.8841(2)$ \\
\hline & & B5 & $0.8653(23.01)(4)$ & $0.9048(-8.97)(2)$ & $0.9065(-10.88)(1)$ & $0.8963(3)$ \\
\hline & & B7 & $0.8736(28.60)(4)$ & $0.9094(0.41)(3)$ & $0.9102(-0.51)(1)$ & $0.9098(2)$ \\
\hline & & Mean & $0.8623(22.31)(4)$ & $0.8983(-5.18)(2)$ & $0.9013(-8.30)(1)$ & $0.8930(3)$ \\
\hline & \multirow{7}{*}{ RMSE (\%) } & B1 & $22.5714(14.34)(4)$ & $18.0438(-7.15)(1)$ & $19.0042(-1.73)(2)$ & $19.3336(3)$ \\
\hline & & B2 & $17.9176(6.98)(4)$ & $15.1464(-10.04)(2)$ & $14.5512(-14.54)(1)$ & $16.6672(3)$ \\
\hline & & B3 & $18.2034(12.59)(4)$ & $15.4631(-2.90)(2)$ & $14.5452(-9.40)(1)$ & $15.9122(3)$ \\
\hline & & B4 & $14.9230(19.43)(4)$ & $11.8142(-1.77)(2)$ & $11.3644(-5.80)(1)$ & $12.0233(3)$ \\
\hline & & B5 & 13.1452 (12.53) (4) & $11.0762(-3.81)(2)$ & $10.7289(-7.17)(1)$ & $11.4985(3)$ \\
\hline & & B7 & $19.5603(14.40)(4)$ & $17.0861(2.01)(3)$ & $16.6975(-0.27)(1)$ & $16.7432(2)$ \\
\hline & & Mean & $17.7202(13.38)(4)$ & $14.7716(-3.94)(2)$ & $14.4819(-6.49)(1)$ & $15.3630(3)$ \\
\hline & SAM & - & $5.0768(15.43)(4)$ & $3.9739(-8.04)(1)$ & $4.0982(-4.76)(2)$ & $4.2934(3)$ \\
\hline & $Q 2^{n}$ & - & $0.5633(11.13)(4)$ & $0.6304(-5.02)(1)$ & $0.5906(5.20)(3)$ & $0.6119(2)$ \\
\hline \multirow{16}{*}{$\begin{array}{c}\text { DOY187 } \\
\text { by } 123\end{array}$} & \multirow{7}{*}{$\mathrm{CC}$} & B1 & $0.5567(24.35)(4)$ & 0.6457 (5.36) (3) & 0.6061 (14.87) (2) & $0.6647(1)$ \\
\hline & & B2 & $0.6287(14.56)(4)$ & 0.6654 (5.17) (3) & $0.6687(4.23)(2)$ & $0.6828(1)$ \\
\hline & & B3 & $0.6461(25.66)(4)$ & 0.6946 (13.87) (3) & 0.7164 (7.24) (2) & $0.7369(1)$ \\
\hline & & B4 & $0.6476(27.93)(3)$ & $0.5835(39.03)(4)$ & $0.7671(-9.07)(1)$ & $0.7460(2)$ \\
\hline & & B5 & $0.6729(21.54)(4)$ & $0.6917(16.75)(3)$ & $0.7396(1.45)(2)$ & $0.7433(1)$ \\
\hline & & B7 & $0.5672(17.12)(4)$ & $0.6345(1.84)(3)$ & $0.6431(-0.51)(1)$ & $0.6413(2)$ \\
\hline & & Mean & $0.6199(21.86)(4)$ & $0.6526(13.67)(3)$ & $0.6902(3.04)(2)$ & $0.7025(1)$ \\
\hline & \multirow{7}{*}{ RMSE (\%) } & B1 & 31.2501 (3.01) (3) & $31.4319(3.57)(4)$ & $27.9180(-8.57)(1)$ & $30.3104(2)$ \\
\hline & & B2 & $24.4620(11.01)(4)$ & $22.2539(2.18)(3)$ & $21.4301(-1.58)(1)$ & $21.7683(2)$ \\
\hline & & B3 & 32.7879 (15.33) (4) & $30.3641(8.57)(3)$ & $28.1812(1.49)(2)$ & $27.7627(1)$ \\
\hline & & B4 & $29.9387(17.66)(3)$ & $32.2695(23.60)(4)$ & $23.7693(-3.72)(1)$ & $24.6530(2)$ \\
\hline & & B5 & $18.4070(13.33)(4)$ & 18.4039 (13.31) (3) & $15.8285(-0.79)(1)$ & $15.9536(2)$ \\
\hline & & B7 & 33.1154 (12.74) (4) & $30.7261(5.95)(3)$ & $27.9871(-3.25)(1)$ & $28.8972(2)$ \\
\hline & & Mean & 28.3268 (12.18) (4) & $27.5749(9.53)(3)$ & $24.1857(-2.74)(1)$ & $24.8909(2)$ \\
\hline & SAM & - & 9.4225 (11.63) (4) & $9.2288(9.78)(3)$ & $8.0472(-3.47)(1)$ & $8.3265(2)$ \\
\hline & $Q 2^{n}$ & - & $0.3603(9.00)(4)$ & $0.4212(-0.58)(1)$ & $0.3613(8.86)(3)$ & $0.4179(2)$ \\
\hline \multirow{16}{*}{$\begin{array}{c}\text { DOY235 } \\
\text { by } 187\end{array}$} & \multirow{7}{*}{$\mathrm{CC}$} & B1 & 0.8409 (17.78) (4) & $0.8466(14.72)(3)$ & $0.8756(-5.17)(1)$ & $0.8692(2)$ \\
\hline & & B2 & $0.8665(20.78)(4)$ & $0.8790(12.60)(3)$ & $0.9003(-6.03)(1)$ & $0.8943(2)$ \\
\hline & & B3 & $0.8981(25.23)(4)$ & $0.9033(21.24)(3)$ & $0.9254(-2.17)(1)$ & $0.9238(2)$ \\
\hline & & B4 & $0.8482(27.39)(4)$ & $0.8522(25.42)(3)$ & $0.8932(-3.26)(1)$ & $0.8898(2)$ \\
\hline & & B5 & $0.8919(21.06)(4)$ & $0.9067(8.49)(3)$ & $0.9215(-8.72)(1)$ & $0.9146(2)$ \\
\hline & & B7 & $0.8731(25.50)(4)$ & 0.8949 (9.98) (3) & $0.9098(-4.82)(1)$ & $0.9054(2)$ \\
\hline & & Mean & $0.8698(22.96)(4)$ & $0.8805(15.41)(3)$ & $0.9043(-5.03)(1)$ & $0.8995(2)$ \\
\hline & \multirow{7}{*}{ RMSE (\%) } & B1 & $25.0868(8.94)(4)$ & $24.2451(5.77)(3)$ & $22.2098(-2.86)(1)$ & $22.8452(2)$ \\
\hline & & B2 & $19.2244(11.34)(4)$ & $17.9257(4.91)(3)$ & $16.4230(-3.79)(1)$ & $17.0448(2)$ \\
\hline & & B3 & $23.5860(13.98)(4)$ & 22.7859 (10.96) (3) & $19.9418(-1.74)(1)$ & $20.2884(2)$ \\
\hline & & B4 & $22.8159(14.81)(3)$ & $22.9792(15.41)(4)$ & $18.6695(-4.11)(1)$ & $19.4376(2)$ \\
\hline & & B5 & $13.8405(10.52)(4)$ & $13.0303(4.95)(3)$ & $11.9447(-3.68)(1)$ & $12.3848(2)$ \\
\hline & & B7 & $24.3245(14.54)(4)$ & $22.0212(5.60)(3)$ & $20.2364(-2.73)(1)$ & $20.7886(2)$ \\
\hline & & Mean & 21.4797 (12.35) (4) & $20.4979(7.94)(3)$ & $18.2375(-3.15)(1)$ & $18.7983(2)$ \\
\hline & SAM & - & $6.9054(10.42)(4)$ & $6.5378(5.38)(3)$ & $6.0875(-1.61)(1)$ & $6.1858(2)$ \\
\hline & $Q 2^{n}$ & - & $0.4593(6.55)(4)$ & $0.4935(0.24)(2)$ & $0.4807(2.71)(3)$ & $0.4947(1)$ \\
\hline
\end{tabular}

For DOY187by123, CC indicates ISTRUM slightly outperforms FSDAF when we compare the correlation between synthesized and observed image. However, RRMSE indicates the image synthesized by FSDAF has smaller difference with observed image than the one synthesized by ISTRUM. The inconsistency between CC and RRMSE is reasonable because CC measures the strength of linear relationship while RRMSE measures the difference of pixel values between observed and synthetic image. The inconsistence was also found in [34]. The change of land surface leads to the reflectance changing at varied magnitudes for different bands, which means a band-wise difference in 
the intensity of temporal change. Since performance of different methods is influenced by factors such as the complexity and intensity of temporal change, spatial heterogeneity and optimal parameters [54], the accuracy is band dependent. In general, the results indicate FSDAF has the best performance in most cases. However, it is also worth noting that FSDAF takes advantages of both spatial-unmixing and weigh-function based methods and predicts land cover type change with a spatial interpolation method which is suitable to deal with small land cover type change [10]. Nonetheless, land cover type change is not specifically considered in STDFA, HCM and ISTRUM, which limits their performance when compared with FSDAF.

Among all methods, accuracy of DOY187by123 is lower than DOY123by107 and DOY235by187 because of the weak correlation between images on DOY123 and DOY187. This result indicates selection of L-C image pair is important for all the compared methods. Accuracy could be improved by selecting L-C pair with high correlation [54].

Table 7 summarizes the accuracy of ESTARFM and ISTRUM for experiments with two L-C pairs. ISTRUM outperformed ESTARFM for some bands because the intensity of temporal change is different for each band. The difference in theoretical basis of ISTRUM and ESTARFM results in their abilities to deal with temporal change of different complexity and intensity being varied. In general, performance of ISTRUM and ESTARFM are comparable because the accuracy values are close for both DOY123by107\&235 and DOY187by107\&235. The maximum difference is $0.0104 \%$ for CC and $0.55 \%$ for RRMSE.

Table 7. Comparison between ESTARFM and ISTRUM.

\begin{tabular}{|c|c|c|c|c|c|}
\hline & & \multicolumn{2}{|c|}{ DOY123By107\&235 } & \multicolumn{2}{|c|}{ DOY187By107\&235 } \\
\hline & & ESTARFM (RRE \%) & ISTRUM & ESTARFM (RRE \%) & ISTRUM \\
\hline \multirow{7}{*}{$\mathrm{CC}$} & B1 & $0.9099(-8.98)$ & 0.9018 & $0.8664(-5.28)$ & 0.8594 \\
\hline & B2 & $0.8957(-0.84)$ & 0.8948 & $0.8895(-7.06)$ & 0.8817 \\
\hline & B3 & 0.9070 (1.81) & 0.9087 & $0.9219(-4.36)$ & 0.9185 \\
\hline & B4 & $0.8222(19.78)$ & 0.8574 & $0.8822(9.67)$ & 0.8936 \\
\hline & B5 & $0.8676(14.17)$ & 0.8864 & $0.8988(12.82)$ & 0.9118 \\
\hline & B7 & 0.8873 (13.75) & 0.9028 & $0.8936(5.85)$ & 0.8998 \\
\hline & Mean & $0.8816(6.62)$ & 0.8920 & $0.8921(1.94)$ & 0.8941 \\
\hline \multirow{7}{*}{ RRMSE (\%) } & B1 & $17.4677(2.47)$ & 17.0360 & $17.0265(-10.44)$ & 18.8046 \\
\hline & B2 & $14.2008(-1.94)$ & 14.4764 & $12.7523(-6.37)$ & 13.5640 \\
\hline & B3 & $14.4847(-0.28)$ & 14.5256 & $15.5686(-3.13)$ & 16.0557 \\
\hline & B4 & 14.1695 (11.65) & 12.5183 & $17.6942(2.41)$ & 17.2672 \\
\hline & B5 & $12.6173(5.17)$ & 11.9652 & $10.6253(4.65)$ & 10.1311 \\
\hline & B7 & $17.6448(4.85)$ & 16.7899 & $16.1206(2.64)$ & 15.6947 \\
\hline & Mean & 15.0975 (3.65) & 14.5519 & $14.9646(-1.71)$ & 15.2529 \\
\hline SAM & - & $4.8290(8.19)$ & 4.4335 & $5.0645(0.68)$ & 5.0299 \\
\hline$Q 2^{n}$ & - & $0.5984(2.12)$ & 0.6069 & $0.5536(-2.37)$ & 0.5430 \\
\hline
\end{tabular}

\subsubsection{Computational Efficiency}

In this study, ISTRUM was implemented in Interactive Data Language (IDL) development environment. The code can be found at URL: https://github.com/JianhangMa/ISTRUM.git. Experiments were run on a PC with an Intel(R) Core(TM) i5-7300HQ 2.50 GHz CPU and 8 GB memory. Average time for each execution is shown in Table 8. ISTRUM is about 32 times faster than HCM, 480 times faster than ESTARFM and 1178 times faster than FSDAF. Computational efficiency of ISTRUM is much higher than ESTARFM and FSDAF. For large-scale and long-term applications of spatiotemporal fusion methods, ISTRUM provides a valuable alternative because it can synthesize images with acceptable accuracy in a short time. 
Table 8. Execution time for STDFA, ISTRUM, HCM, ESTARFM and FSDAF. s, $\mathrm{m}$, and h denote second, minute, and hour, respectively.

\begin{tabular}{cccccc}
\hline & STDFA & ISTRUM & HCM & ESTARFM & FSDAF \\
\hline Time & $15.387 \mathrm{~s}$ & $19.025 \mathrm{~s}$ & $10.25 \mathrm{~m}$ & $2.54 \mathrm{~h}$ & $6.22 \mathrm{~h}$ \\
\hline
\end{tabular}

\section{Discussion}

Spatiotemporal image fusion is a feasible and cost-efficient approach to synthesize images with high spatial and temporal resolution [15]. Spatial-unmixing based method is one kind of the most widely studied spatiotemporal fusion methods $[6,25,26,40,41]$. One challenge is that the image derived in spatial-unmixing based method generally lacks spectral variability and spatial details $[10,26,27]$. To tackle the problem, this study improved the STRUM, which is a well-performing spatial-unmixing based method [26], by applying fine-resolution abundance image. Experimental results revealed ISTRUM generated higher accuracy than STRUM and was competitive with benchmark spatiotemporal fusion methods. Based on the results in this study, relevant issues are discussed in the following paragraphs.

Used as the base images in spatiotemporal fusion methods, L-C image pair is significant to improve the accuracy of synthetic image. Accuracy of the same method varied greatly when input L-C pair is different. In our study, Landsat-like image on DOY187 synthesized based on L-C pair on DOY235 (Section 4.4) has higher accuracy than the one based on DOY123 and DOY107. The accuracy of Landsat-like image is improved when L-C image pair strongly correlates to images on $T_{P}$. Cheng et al. [28] found accuracy increased as the time interval between $T_{B}$ and $T_{P}$ decreased because the likelihood of large temporal change is low and correlation between images on $T_{B}$ and $T_{P}$ is high when the time interval is short. According to the correlation between coarse-resolution images on $T_{B}$ and $T_{P}$, optimal L-C pair can be determined [54]. In addition, multiple L-C pairs can also improve the accuracy for situations when more than one L-C pair are available.

Since accuracy of spatiotemporal fusion methods is influenced by spatial heterogeneity [5,7], land cover type [48], and the complexity of temporal change [38], performance of the methods varied under different conditions. There is no universal method that performs well under all conditions [34]. For example, although FSDAF considers land cover type change, it has problems in overestimating spatial variations [11]; and ESTARFM improves the accuracy in heterogeneous regions but has problems in estimating nonlinear reflectance changes [38]. Our study also found ISTRUM outperformed HCM when temporal change was large while HCM was better when temporal change was small. Therefore, selecting and combining different methods according to the conditions is meaningful in application [34]. For example, to synthesize time series NDVI, methods with better performance at Red and NIR bands are preferred considering the fusion accuracy is band dependent. In addition, for large area and long term applications of spatiotemporal fusion methods, processing many images is required. The trade-off between accuracy and computational efficiency should be considered. With one user defined parameter and high computational efficiency, ISTRUM provides an easy-to-use and efficient alternative.

Fine- and coarse-resolution images are generally regarded as consistent when developing the spatiotemporal fusion methods $[10,11]$. However, sensor difference commonly existed and impacts fusion accuracy. Xie et al. [54] found MODIS images with smaller view zenith angle produce better predictions. Preprocessing to reduce of sensor difference helps to improve the accuracy. Models to adjust sensor difference are worth of integration into spatiotemporal fusion methods. Similar with previous studies [47,48], ISTRUM adjusted the sensor difference with a simple linear model and improved the performance to some degree. However, nonlinear models [46] to normalize the inconsistency between fine- and coarse-resolution images are worth studying in the future. 


\section{Conclusions}

To tackle the shortcomings of STRUM, this study proposed ISTRUM to improve STRUM from three aspects: (1) apply fine-resolution abundance image rather than hard-classification image in the spatial-unmixing process; (2) adjust sensor difference between fine- and coarse-resolution images; and (3) strengthen the applicability when multiple L-C image pairs are utilized. Experimental results demonstrated that:

(1) All three improvements contribute to improving performance of ISTRUM when compared with STRUM. ISTRUM is robust to endmember variability, and the spectra of Global SVD endmembers could be directly applied. Sliding-window size is the only parameter that needs to be defined by the user and decreases the accuracy of ISTRUM when it is large.

(2) The selection of L-C image pair plays a significant role in the fusion methods. Accuracy is improved when the L-C image pair is strongly correlated with the image on prediction date.

(3) Performance of the fusion methods are different under different conditions. Selecting and combining different methods according to the conditions is meaningful in application. ISTRUM is an easy-to-use and efficient alternative to synthesize time series of Landsat-like images on a global scale.

Author Contributions: W.Z. and B.Z. developed the algorithm and conceived the experiments; J.M. developed the codes, performed the experiments and wrote the manuscript; and A.M. and L.G. structured and edited the manuscript.

Funding: This research was funded by the National Key R\&D Program of China (2016YFB0500304) and the National Natural Science Foundation of China (91638201).

Acknowledgments: The authors would like to thank I. Emelyanova for sharing the Landsat and MODIS data of the study area, and thank C. Small for sharing the spectra of Global SVD Endmembers. They would also like to thank the anonymous reviewers for their insightful comments and suggestions, which helped to improve the quality of the manuscript significantly. Thanks to Kwan C. and Zhu X. for the free accesses to their well-developed processing program HCM and FSDAF.

Conflicts of Interest: The authors declare no conflict of interest.

\section{References}

1. Wulder, M.A.; White, J.C.; Loveland, T.R.; Woodcock, C.E.; Belward, A.S.; Cohen, W.B.; Fosnight, E.A.; Shaw, J.; Masek, J.G.; Roy, D.P. The global Landsat archive: Status, consolidation, and direction. Remote Sens. Environ. 2016, 185, 271-283. [CrossRef]

2. Fisher, J.I.; Mustard, J.F.; Vadeboncoeur, M.A. Green leaf phenology at Landsat resolution: Scaling from the field to the satellite. Remote Sens. Environ. 2006, 100, 265-279. [CrossRef]

3. Zheng, B.; Myint, S.W.; Thenkabail, P.S.; Aggarwal, R.M. A support vector machine to identify irrigated crop types using time-series Landsat NDVI data. Int. J. Appl. Earth Obs. Geoinf. 2015, 34, 103-112. [CrossRef]

4. Kovalskyy, V.; Roy, D. The global availability of Landsat $5 \mathrm{TM}$ and Landsat 7 ETM+ land surface observations and implications for global 30m Landsat data product generation. Remote Sens. Environ. 2013, 130, $280-293$. [CrossRef]

5. Gao, F.; Masek, J.; Schwaller, M.; Hall, F. On the blending of the Landsat and MODIS surface reflectance: Predicting daily Landsat surface reflectance. IEEE Trans. Geosci. Remote Sens. 2006, 44, 2207-2218.

6. Zurita-Milla, R.; Kaiser, G.; Clevers, J.; Schneider, W.; Schaepman, M. Downscaling time series of MERIS full resolution data to monitor vegetation seasonal dynamics. Remote Sens. Environ. 2009, 113, 1874-1885. [CrossRef]

7. Zhu, X.; Chen, J.; Gao, F.; Chen, X.; Masek, J.G. An enhanced spatial and temporal adaptive reflectance fusion model for complex heterogeneous regions. Remote Sens. Environ. 2010, 114, 2610-2623. [CrossRef]

8. Huang, B.; Song, H. Spatiotemporal Reflectance Fusion via Sparse Representation. IEEE Trans. Geosci. Remote Sens. 2012, 50, 3707-3716. [CrossRef]

9. Song, H.; Huang, B. Spatiotemporal Satellite Image Fusion through One-Pair Image Learning. IEEE Trans. Geosci. Remote Sens. 2013, 51, 1883-1896. [CrossRef] 
10. Zhu, X.; Helmer, E.H.; Gao, F.; Liu, D.; Chen, J.; Lefsky, M.A. A flexible spatiotemporal method for fusing satellite images with different resolutions. Remote Sens. Environ. 2016, 172, 165-177. [CrossRef]

11. Wang, Q.; Atkinson, P.M. Spatio-temporal fusion for daily Sentinel-2 images. Remote Sens. Environ. 2018, 204, 31-42. [CrossRef]

12. Zhao, Y.; Huang, B.; Song, H. A robust adaptive spatial and temporal image fusion model for complex land surface changes. Remote Sens. Environ. 2018, 208, 42-62. [CrossRef]

13. Yue, L.; Shen, H.; Li, J.; Yuan, Q.; Zhang, H.; Zhang, L. Image super-resolution: The techniques, applications, and future. Signal Process. 2016, 128, 389-408. [CrossRef]

14. Shen, H.; Meng, X.; Zhang, L. An Integrated Framework for the Spatio-Temporal-Spectral Fusion of Remote Sensing Images. IEEE Trans. Geosci. Remote Sens. 2016, 54, 7135-7148. [CrossRef]

15. Zhu, X.; Cai, F.; Tian, J.; Williams, T.K.A. Spatiotemporal Fusion of Multisource Remote Sensing Data: Literature Survey, Taxonomy, Principles, Applications, and Future Directions. Remote Sens. 2018, 10, 527.

16. Hilker, T.; Wulder, M.A.; Coops, N.C.; Seitz, N.; White, J.C.; Gao, F.; Masek, J.G.; Stenhouse, G. Generation of dense time series synthetic Landsat data through data blending with MODIS using a spatial and temporal adaptive reflectance fusion model. Remote Sens. Environ. 2009, 113, 1988-1999. [CrossRef]

17. Gao, F.; Hilker, T.; Zhu, X.; Anderson, M.; Masek, J.; Wang, P.; Yang, Y. Fusing Landsat and MODIS Data for Vegetation Monitoring. IEEE Geosci. Remote Sens. Mag. 2015, 3, 47-60. [CrossRef]

18. Zhu, L.; Radeloff, V.C.; Ives, A.R. Improving the mapping of crop types in the Midwestern U.S. by fusing Landsat and MODIS satellite data. Int. J. Appl. Earth Obs. Geoinf. 2017, 58, 1-11. [CrossRef]

19. Weng, Q.; Fu, P.; Gao, F. Generating daily land surface temperature at Landsat resolution by fusing Landsat and MODIS data. Remote Sens. Environ. 2014, 145, 55-67. [CrossRef]

20. Wu, P.; Shen, H.; Zhang, L.; Göttsche, F.M. Integrated fusion of multi-scale polar-orbiting and geostationary satellite observations for the mapping of high spatial and temporal resolution land surface temperature. Remote Sens. Environ. 2015, 156, 169-181. [CrossRef]

21. Cammalleri, C.; Anderson, M.C.; Gao, F.; Hain, C.R.; Kustas, W.P. A data fusion approach for mapping daily evapotranspiration at field scale. Water Resour. Res. 2013, 49, 4672-4686. [CrossRef]

22. Yang, Y.; Anderson, M.C.; Gao, F.; Hain, C.R.; Semmens, K.A.; Kustas, W.P.; Noormets, A.; Wynne, R.H.; Thomas, V.A.; Sun, G. Daily Landsat-scale evapotranspiration estimation over a forested landscape in North Carolina, USA, using multi-satellite data fusion. Hydrol. Earth Syst. Sci. 2017, 21, 1017-1037. [CrossRef]

23. Chang, N.B.; Vannah, B. Monitoring the total organic carbon concentrations in a lake with the integrated data fusion and machine-learning (IDFM) technique. In Proceedings of the Remote Sensing and Modeling of Ecosystems for Sustainability, San Diego, CA, USA, 12-16 August 2012.

24. Swain, R.; Sahoo, B. Mapping of heavy metal pollution in river water at daily time-scale using spatio-temporal fusion of MODIS-aqua and Landsat satellite imageries. J. Environ. Manag. 2017, 192, 1-14. [CrossRef] [PubMed]

25. Wu, M.; Niu, Z.; Wang, C.; Wu, C.; Wang, L. Use of MODIS and Landsat time series data to generate high-resolution temporal synthetic Landsat data using a spatial and temporal reflectance fusion model. J. Appl. Remote Sens. 2012, 6, 063507.

26. Gevaert, C.M.; García-Haro, F.J. A comparison of STARFM and an unmixing-based algorithm for Landsat and MODIS data fusion. Remote Sens. Environ. 2015, 156, 34-44. [CrossRef]

27. Xu, Y.; Huang, B.; Xu, Y.; Cao, K.; Guo, C.; Meng, D. Spatial and Temporal Image Fusion via Regularized Spatial Unmixing. IEEE Geosci. Remote Sens. Lett. 2015, 12, 1362-1366.

28. Cheng, Q.; Liu, H.; Shen, H.; Wu, P.; Zhang, L. A Spatial and Temporal Nonlocal Filter-Based Data Fusion Method. IEEE Trans. Geosci. Remote Sens. 2017, 55, 4476-4488. [CrossRef]

29. Boyte, S.P.; Wylie, B.K.; Rigge, M.B.; Dahal, D. Fusing MODIS with Landsat 8 data to downscale weekly normalized difference vegetation index estimates for central Great Basin rangelands, USA. GISci. Remote Sens. 2018, 55, 376-399. [CrossRef]

30. Tan, Z.; Yue, P.; Di, L.; Tang, J. Deriving High Spatiotemporal Remote Sensing Images Using Deep Convolutional Network. Remote Sens. 2018, 10, 1066. [CrossRef]

31. Chen, B.; Huang, B.; Xu, B. Comparison of Spatiotemporal Fusion Models: A Review. Remote Sens. 2015, 7, 1798-1835. [CrossRef]

32. Hazaymeh, K.; Hassan, Q.K. Fusion of MODIS and Landsat-8 Surface Temperature Images: A New Approach. PLoS ONE 2015, 10, e0117755. [CrossRef] [PubMed] 
33. Hazaymeh, K.; Hassan, Q.K. Spatiotemporal image-fusion model for enhancing the temporal resolution of Landsat-8 surface reflectance images using MODIS images. J. Appl. Remote Sens. 2015, 9, 096095. [CrossRef]

34. Kwan, C.; Budavari, B.; Gao, F.; Zhu, X. A Hybrid Color Mapping Approach to Fusing MODIS and Landsat Images for Forward Prediction. Remote Sens. 2018, 10, 520. [CrossRef]

35. Kwan, C.; Zhu, X.; Gao, F.; Chou, B.; Perez, D.; Li, J.; Shen, Y.; Koperski, K.; Marchisio, G. Assessment of Spatiotemporal Fusion Algorithms for Planet and Worldview Images. Sensors 2018, 18, 1051. [CrossRef] [PubMed]

36. Xue, J.; Leung, Y.; Fung, T. A Bayesian Data Fusion Approach to Spatio-Temporal Fusion of Remotely Sensed Images. Remote Sens. 2017, 9, 1310. [CrossRef]

37. Xie, D.; Zhang, J.; Zhu, X.; Pan, Y.; Liu, H.; Yuan, Z.; Yun, Y. An Improved STARFM with Help of an Unmixing-Based Method to Generate High Spatial and Temporal Resolution Remote Sensing Data in Complex Heterogeneous Regions. Sensors 2016, 16, 207. [CrossRef] [PubMed]

38. Emelyanova, I.V.; McVicar, T.R.; Niel, T.G.V.; Li, L.T.; van Dijk, A.I. Assessing the accuracy of blending Landsat-MODIS surface reflectances in two landscapes with contrasting spatial and temporal dynamics: A framework for algorithm selection. Remote Sens. Environ. 2013, 133, 193-209. [CrossRef]

39. Small, C. The Landsat ETM+ spectral mixing space. Remote Sens. Environ. 2004, 93, 1-17. [CrossRef]

40. Busetto, L.; Meroni, M.; Colombo, R. Combining medium and coarse spatial resolution satellite data to improve the estimation of sub-pixel NDVI time series. Remote Sens. Environ. 2008, 112, 118-131. [CrossRef]

41. Amorós-López, J.; Gómez-Chova, L.; Alonso, L.; Guanter, L.; Moreno, J.; Camps-Valls, G. Regularized Multiresolution Spatial Unmixing for ENVISAT/MERIS and Landsat/TM Image Fusion. IEEE Geosci. Remote Sens. Lett. 2011, 8, 844-848. [CrossRef]

42. Ma, W.K.; Bioucas-Dias, J.M.; Chan, T.H.; Gillis, N.; Gader, P.; Plaza, A.J.; Ambikapathi, A.; Chi, C.Y. A Signal Processing Perspective on Hyperspectral Unmixing: Insights from Remote Sensing. IEEE Signal Process. Mag. 2014, 31, 67-81. [CrossRef]

43. Tobler, W.R. A Computer Movie Simulating Urban Growth in the Detroit Region. Econ. Geogr. 1970, 46, 234-240. [CrossRef]

44. Steven, M.D.; Malthus, T.J.; Baret, F.; Xu, H.; Chopping, M.J. Intercalibration of vegetation indices from different sensor systems. Remote Sens. Environ. 2003, 88, 412-422. [CrossRef]

45. Zhang, H.K.; Roy, D.P.; Yan, L.; Li, Z.; Huang, H.; Vermote, E.; Skakun, S.; Roger, J.C. Characterization of Sentinel-2A and Landsat-8 top of atmosphere, surface, and nadir BRDF adjusted reflectance and NDVI differences. Remote Sens. Environ. 2018, 215, 482-494. [CrossRef]

46. Sadeghi, V.; Ebadi, H.; Ahmadi, F.F. A new model for automatic normalization of multitemporal satellite images using Artificial Neural Network and mathematical methods. Appl. Math. Model. 2013, 37, 6437-6445. [CrossRef]

47. Shen, H.; Wu, P.; Liu, Y.; Ai, T.; Wang, Y.; Liu, X. A spatial and temporal reflectance fusion model considering sensor observation differences. Int. J. Remote Sens. 2013, 34, 4367-4383. [CrossRef]

48. Wu, M.; Wu, C.; Huang, W.; Niu, Z.; Wang, C.; Li, W.; Hao, P. An improved high spatial and temporal data fusion approach for combining Landsat and MODIS data to generate daily synthetic Landsat imagery. Inf. Fusion 2016, 31, 14-25. [CrossRef]

49. Small, C.; Milesi, C. Multi-scale standardized spectral mixture models. Remote Sens. Environ. 2013, 136, 442-454. [CrossRef]

50. Sousa, D.; Small, C. Global cross-calibration of Landsat spectral mixture models. Remote Sens. Environ. 2017, 192, 139-149. [CrossRef]

51. Heinz, D.C.; Chang, C.I. Fully constrained least squares linear spectral mixture analysis method for material quantification in hyperspectral imagery. IEEE Trans. Geosci. Remote Sens. 2001, 39, 529-545. [CrossRef]

52. Amorós-López, J.; Gómez-Chova, L.; Alonso, L.; Guanter, L.; Zurita-Milla, R.; Moreno, J.; Camps-Valls, G. Multitemporal fusion of Landsat/TM and ENVISAT/MERIS for crop monitoring. Int. J. Appl. Earth Obs. Geoinf. 2013, 23, 132-141. [CrossRef]

53. Lasdon, L.S.; Waren, A.D.; Jain, A.; Ratner, M. Design and Testing of a Generalized Reduced Gradient Code for Nonlinear Programming. ACM Trans. Math. Softw. 1978, 4, 34-50. [CrossRef]

54. Xie, D.; Gao, F.; Sun, L.; Anderson, M. Improving Spatial-Temporal Data Fusion by Choosing Optimal Input Image Pairs. Remote Sens. 2018, 10, 1142. [CrossRef] 
55. Wu, J.; Cheng, Q.; Li, H.; Wu, P.; Shen, H. Applicability Analysis of Mono- and Bi-temporal Auxiliary Data in Remote Sensing Spatiotemporal Fusion. Geog. Geo-Inf. Sci. 2017, 5, 9-15.

56. Jarihani, A.A.; McVicar, T.R.; Van Niel, T.G.; Emelyanova, I.V.; Callow, J.N.; Johansen, K. Blending Landsat and MODIS Data to Generate Multispectral Indices: A Comparison of "Index-then-Blend" and "Blend-then-Index" Approaches. Remote Sens. 2014, 6, 9213-9238. [CrossRef]

57. Somers, B.; Asner, G.P.; Tits, L.; Coppin, P. Endmember variability in Spectral Mixture Analysis: A review. Remote Sens. Environ. 2011, 115, 1603-1616. [CrossRef]

58. Ma, J.; Zhang, W.; Marinoni, A.; Gao, L.; Zhang, B. Performance assessment of ESTARFM with different similar-pixel identification schemes. J. Appl. Remote Sens. 2018, 12, 025017. [CrossRef]

59. Garzelli, A.; Nencini, F. Hypercomplex Quality Assessment of Multi/Hyperspectral Images. IEEE Geosci. Remote Sens. Lett. 2009, 6, 662-665. [CrossRef]

60. Wang, Q.; Shi, W.; Atkinson, P.M.; Zhao, Y. Downscaling MODIS images with area-to-point regression kriging. Remote Sens. Environ. 2015, 166, 191-204. [CrossRef]

(c) 2016 by the authors. Licensee MDPI, Basel, Switzerland. This article is an open access article distributed under the terms and conditions of the Creative Commons Attribution (CC BY) license (http://creativecommons.org/licenses/by/4.0/). 